\title{
A taxonomy and evaluation for developing 802.11-based wireless mesh network testbeds
}

\author{
Suleyman Uludag ${ }^{1, *, \dagger}$, Tom Imboden ${ }^{2}$ and Kemal Akkaya ${ }^{3}$ \\ ${ }^{1}$ Department of Computer Science, Engineering \& Physics, University of Michigan-Flint, Flint, MI 48502, USA \\ ${ }^{2}$ School of Information Systems and Applied Technologies, Southern Illinois University Carbondale, Carbondale, \\ IL 62901, USA \\ ${ }^{3}$ Department of Computer Science, Southern Illinois University Carbondale, Carbondale, IL 62901, USA
}

\begin{abstract}
SUMMARY
The definition of wireless mesh networks (WMNs) has been used in the literature to connote and epitomize the ideal, ubiquitous, pervasive, and autonomic networking technology. An increasing interest has been emerging on the development of 802.11-based WMN testbeds to test the new ideas and approaches more realistically as opposed to relying solely on simulations. Although the developed testbeds have provided several insights to researchers for furthering the technology, there are still several issues that need to be addressed, particularly, with the approval of new standards, such as IEEE 802.11s, IEEE 802.11n, and IEEE 802.16, and upcoming protocols, such as IEEE 802.11ac, 802.11ad, 802.11ah, and 802.11af TV White Space efforts. In this paper, our goal is to provide a taxonomy and insightful guidelines for the creation of 802.11based WMN testbeds as well as to identify several features that future WMN testbeds should possess. Utilizing these features, we evaluate the existing WMN testbeds. Finally, in addition to the existing WMN testbed experiments conducted at several layers of the protocol stack, we provide a list of open future research issues that can benefit from experiments on WMN testbeds. Copyright @ 2011 John Wiley \& Sons, Ltd.
\end{abstract}

Received 19 November 2010; Revised 21 January 2011; Accepted 26 April 2011

KEY WORDS: wireless mesh networks; testbeds

\section{INTRODUCTION}

Wireless computing and communications devices have permeated every aspect of business and personal lives. With the increasing interest and developments in wireless technologies [1-5], wireless mesh networks (WMNs) have started to emerge as the most promising model for integrating different wireless networks and for providing broadband access, availability, and coverage to various wireless clients. WMNs are usually touted in the literature as a concept network with a rich set of desirable features and capabilities. They are typically capable of self-organizing, self-healing, selfconfiguring, and self-maintaining, while providing high availability, resilience, and fault-tolerance low cost [6-8].

Wireless mesh networks have their own features and characteristics that distinguish them from Mobile Ad hoc NETworks (MANETs), wireless sensor networks (WSNs), and wireless local area networks (LANs). For instance, the mobility and power consumption is not much of a concern in WMNs because the mesh routers usually do not move, and they have power supplies. Only the mesh clients can move partially and may have limited power supplies. In addition, WMNs can have clients and routers with multiple radios/channels. Routing with multiple channels can provide better

\footnotetext{
*Correspondence to: Suleyman Uludag, Department of Computer Science, Engineering \& Physics, University of Michigan-Flint, Flint, MI 48502, USA.

†E-mail: uludag@umich.edu 
coverage and throughput to boost the performance of voice over Internet Protocol (VoIP), multimedia teleconferencing, and video-on-demand applications that require higher bandwidth and lower end-to-end delays. We provide a more comprehensive discussion on WMNs and key underlying characteristics in Section 2.

Within the last decade, there has been a lot of research on WMNs. This research has mainly focused on routing and medium access control (MAC) protocols, channel assignment, QoS provisioning, and interference avoidance [6]. In these research efforts, the performance of the proposed protocols on the aforementioned topics has been widely tested in network simulators, such as NS-2 [9], NS-3 [10], QualNet (Scalable Network Technologies, Inc., Los Angeles, CA, USA) [11], Glomosim [12], OPNET (OPNET Technologies, Inc., Bethesda, MD, USA) [13], and JSim [14]. Although this approach works to some extent, the performance evaluations may not be applicable to real-life wireless networks because of several unrealistic assumptions within the simulations, and the inability of the simulators to accurately reflect the behavior of the wireless environment [15]. Inaccuracy of lower-layer modeling, especially of the physical layer, such as propagation environment, interface characteristics, and path loss parameters, are elaborated in [16] and [17]. The authors further noted that granularity in simulations is at packet-level, whereas the theoretical analysis these simulations are based on use bit or symbol-level derivations. Several studies, such as $[18,19]$ note the deficiency of unit disk graph ${ }^{*}$ assumption to model the topology of the wireless networks. [19] includes transceiver properties, propagation model, radio link model, interference model, and energy consumption parameters as part of the unrealistic assumptions in wireless simulations. Inadequacy of simplistic mobility model and radio wave propagation characteristics in wireless simulations are explained in [20]. Imprecision of multichannel interference modeling is discussed in [21]. Inaccuracy of neighborhood discovery and radio link symmetry in simulations are described in [22]. Negative impact of incorrect physical layer assumptions, such as signal reception, path loss, fading, interference, noise computation, and preamble length, lead to false performance evaluation at higher layers [23]. After studying about 2200 published results, authors in [15] identified six unrealistic assumptions in wireless simulations as follows:

(1) The world is flat.

(2) The transmission area of a radio is circular.

(3) All radios have equal range.

(4) If I can hear you, you can hear me (symmetry).

(5) If I can hear you at all, I can hear you perfectly.

(6) Signal strength is a simple function of distance.

Random waypoint model, symmetric links, independence from ground height and constant radio links are highlighted in [24] as assumptions that are very far from reality. No message collisions and one routing task at a time methodology are the other problematic areas in wireless simulations as noted in [18].

As a result of the aforementioned problems, prototyping efforts in WMNs have gained increasing attention and interest in the last few years. Because of the decreasing costs and wide availability of wireless equipment, the testbeds are preferred by many researchers to further the study of WMNs as opposed to simulators. In what follows, we use testbeds to refer to WMN testbeds as well as to WMN deployments in several real-life applications.

Although these testbeds have provided valuable insight and experience for the researchers, sharing of the experience with other researchers and interested parties both in academia and industry is crucial and conducive to accelerating future research and validation in a more standardized manner. In particular, with the dynamic nature and constant evolution of new wireless standards and protocols, such as IEEE 802.11s, 802.11ac, 802.11ad, 802.11ah, and 802.16 together with new application areas, such as smart grid, there is a need to analyze the existing testbeds for facilitating the development of effective, efficient, and low-cost testbeds in the future. In this paper, we provide a taxonomy, an analysis, and a discussion of the existing testbeds for those interested in developing or deploying an 802.11-based WMN testbed for their needs. Our main contributions are as follows:

†Unit disk graph model considers a uniform, symmetric, and circular transmission range. 
(1) We provide a list of design choices exploited by the current testbeds for the benefit of new testbed designers. Examining the plethora of available testbed design choices can provide critical insight and conceptual and strategic know-how about which testbed, wireless technology, or protocol might be particularly well suited for a specific development or application environment.

(2) We present a taxonomy of the testbeds with respect to their research goals.

(3) We come up with a list of desirable features and evaluate the existing testbeds with respect to these features.

(4) We provide several future works and experiments that need to be tested under WMN testbeds.

(5) We discuss the features that need to be supported by the future WMN testbeds. Our goal is to help standardize the WMN testbed creation efforts and facilitate the real-life deployments of WMNs with reduced cost and better performance. Further, we would like to promote the use of such testbeds for a variety of problems that have not been tested under realistic environments to increase the accuracy and quality of the research efforts on WMNs.

The rest of the paper is organized as follows. Section 2 provides a brief background on WMNs, a taxonomy of WMNs by means of access method, and an overview of the upcoming IEEE $802.11 \mathrm{~s}$ standard. Section 3 analyzes the alternatives in developing WMN testbeds and provides taxonomies in terms of the design options and research goals. Desirable features of WMN testbeds of the future together with an analysis of the existing testbeds from the same perspective are discussed in Section 4. In Section 5, an examination of major open research issues that can benefit from experimentation on WMN testbeds is presented. Remarks in Section 6 concludes the paper.

\section{BACKGROUND ON WIRELESS MESH NETWORKS}

In this section, we provide a detailed background on several aspects of WMNs that are relevant and pertinent to developing and evaluating WMN testbeds.

\subsection{Architecture and applications}

Wireless mesh networks seem to be the best facilitator for helping users to be always online, anywhere, anytime. WMNs are inherently based on the multihop paradigm and comprise two types of nodes: mesh routers to provide the connectivity and mesh clients to make use of the service provided by the mesh routers for network access. Mesh routers can be built based on general-purpose laptops, desktops, or on dedicated systems, as shown in Figure 1. The mesh client, on the other hand, can be a laptop, desktop, personal digital assistant, cell phone, pager, netbook, tablet, smartphone, or other devices that may or may not be mobile.

A typical architecture of WMNs is shown in Figure 2, where dashed and solid lines denote wireless and wired links, respectively. Mesh routers form a fully connected network, and some provide gateway functionality to the Internet on behalf of the others. A variety of clients and

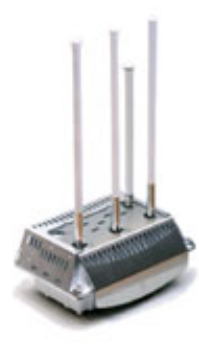

(a)

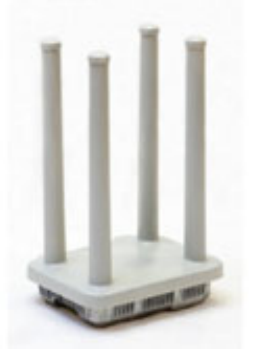

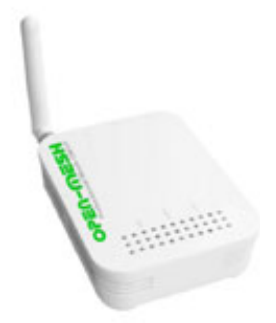

(b)

Figure 1. Examples of mesh routers: (a) Tropos Networks (Tropos Networks Inc., Sunnywale, CA, USA) [25] and (b) Open-Mesh (Open-Mesh Inc., Portland, OR, USA) [26]. 


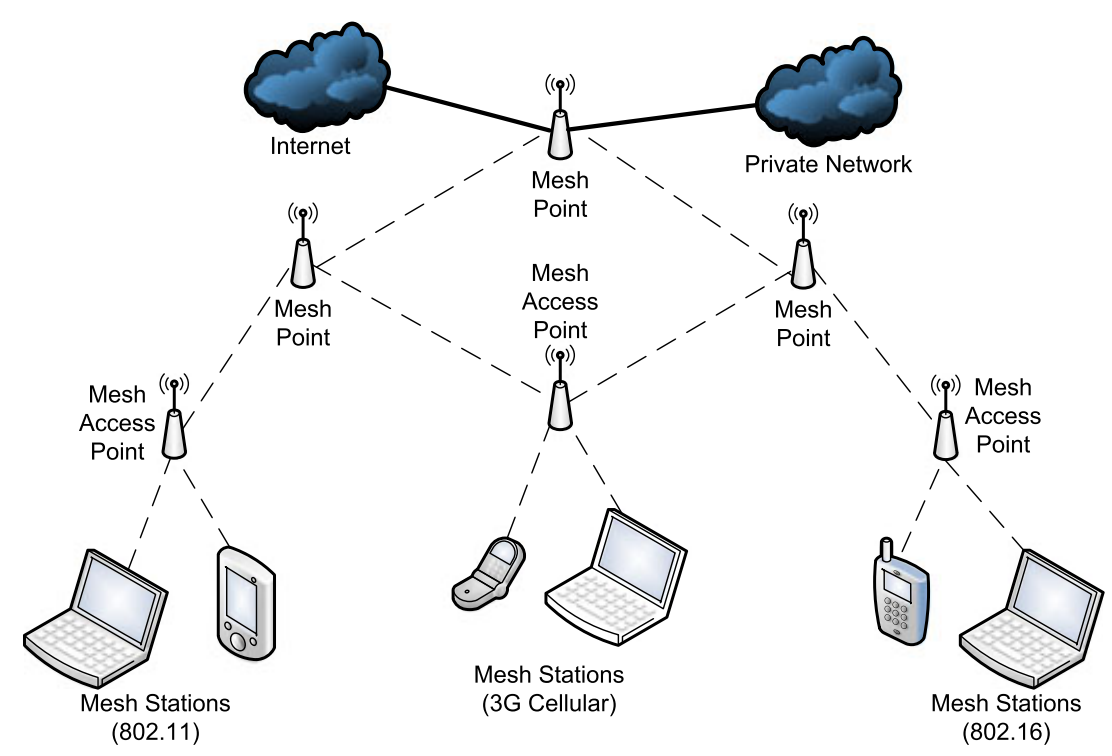

Figure 2. A typical wireless mesh network architecture.

smaller WMNs may form the client base. Although Figure 2 depicts a typical, infrastructure WMN, infrastructureless or peer-to-peer and hybrid architectures are also possible.

Wireless mesh networks have initially been built by using a single channel on a single radio for both the mesh routers and mesh clients. However, theoretical upper bounds of the capacity under single-channel wireless networks is quite limited, that is, $O(1 / \sqrt{n})[27,28]$. Multiple radios, multiple channels, multiple antennas, and/or multiple wireless network cards can provide better coverage, scalability, reliability, and throughput that can boost the performance of many applications, including VoIP, multimedia teleconferencing, and video-on-demand applications that require higher bandwidth and lower end-to-end delays.

Critical applications that can benefit from WMNs are public safety networks [29] ${ }^{\S}$, crisis management [31], first responder networks [32], emergency services [33], wireless community networks [34] (Figure 3(a)), metropolitan area networking (Figure 3(b)), enterprise networking (Figure 3(c)), wireless building automation (Figure 3(d)), intervehicular and intravehicular communications [35] (Figure 3(e)), home networking (Figure 3(f)), neighborhood gaming, remote surveillance, and disaster management.

\subsection{Taxonomy of wireless mesh networks based on medium access methods}

There are many different ways of setting up a WMN. Figure 4 shows one classification of possible alternatives to design a WMN based on the access control mechanisms employed at the MAC layer. Two broad categories are reservation-based and random access. The former regulates the access to the shared medium by means of a pre-arranged mechanism. The latter operates by means of a contention resolution protocol, mainly a variant of carrier sense multiple access/collision avoidance (CSMA/CA). IEEE $802.11 \mathrm{a} / \mathrm{b} / \mathrm{g} / \mathrm{n}$-based proprietary or 802.11 s-based standardized WMNs are the two examples for the random access category. The IEEE $802.11 \mathrm{~s}$ standard is being developed by the IEEE 802.11s Working Group and will be discussed in more details in Section 2.3. Another possibility for deploying a random access WMN is IEEE 802.15.4-based ZigBee protocol stack [36], standardized by the ZigBee Alliance. Unlike the other two, which are focused on LAN/metropolitan

\footnotetext{
${ }^{\S}$ The SAFECOM program Statement of Requirements defines several public safety communications [30] systems, two of which are of particular relevance for this study: incident area network and jurisdictional area network. The former is defined as a temporary network created to support communications during an incident. The latter is defined as a permanent infrastructure covering a wide area, such as city, for supporting communications during an incident.
} 


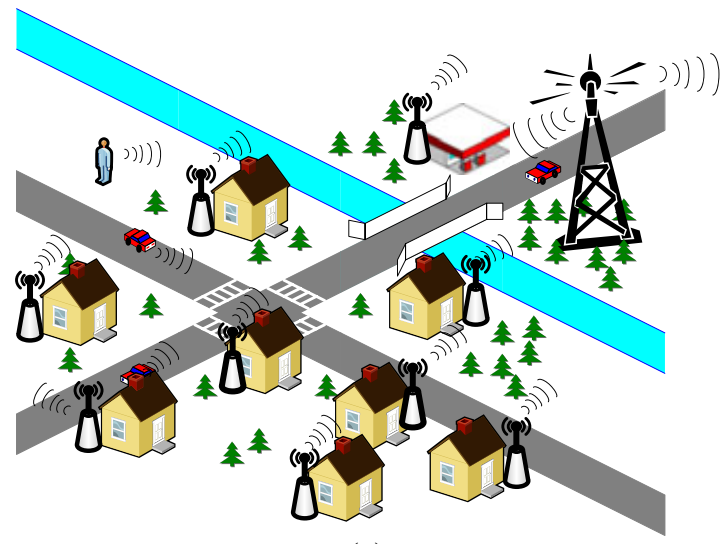

(a)

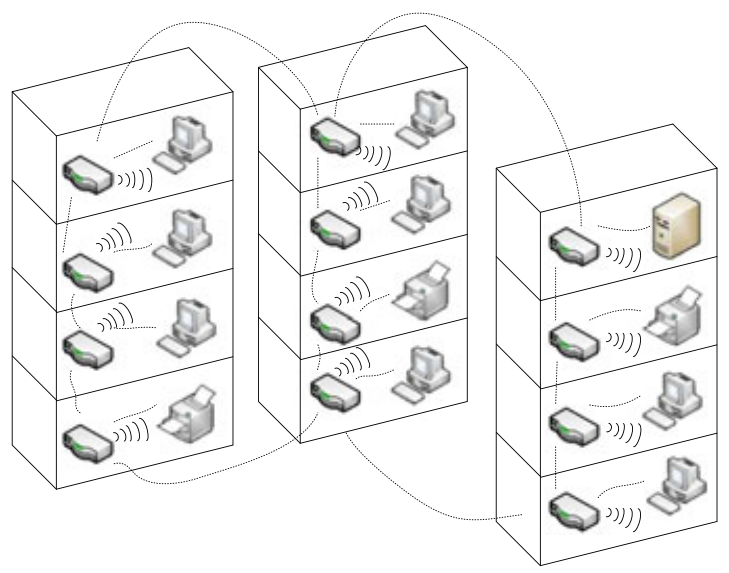

(c)

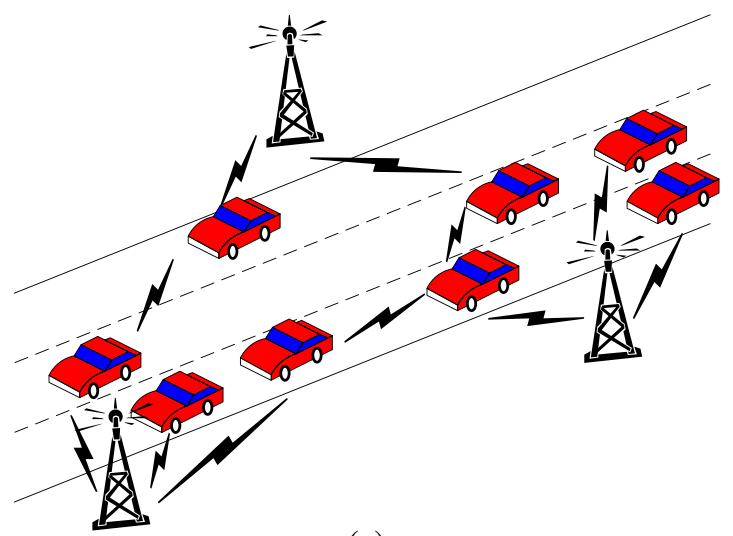

(e)

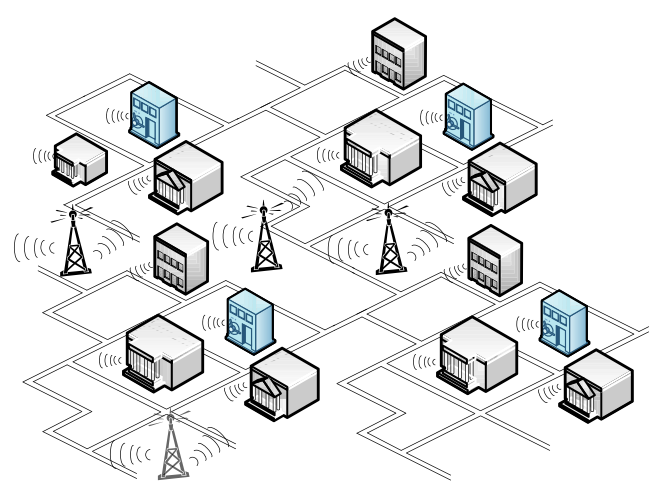

(b)

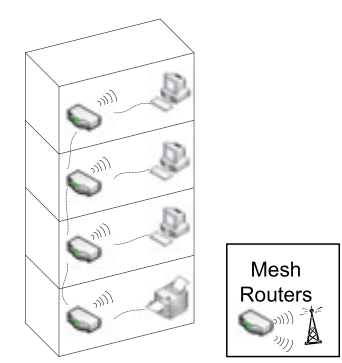

(d)

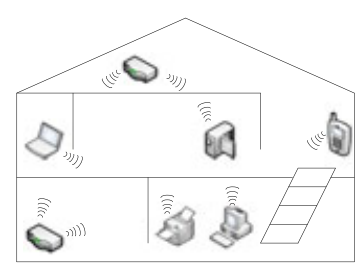

(f)

Figure 3. Some applications of wireless mesh networks: (a) community networking, (b) metropolitan area networking, (c) enterprise networking, (d) wireless building automation, (e) intelligent transportation system, (f) home networking.

area network, ZigBee is proposed for wireless personal area networks (WPANs) as a low-cost and low-power monitoring and control technology based on an open global standard.

Under the reservation-based category, one possibility is based on the code division multiple access (CDMA) method, similar to its usage in cellular networks. IEEE 802.16a-based Worldwide Interoperability for Microwave Access (WiMAX) [37] is a metropolitan area network wireless broadband standard based on the time division multiple access (TDMA) mechanism. A mobile multihop relay operation mode is being developed under the IEEE 802.16j Task Group to improve the performance 


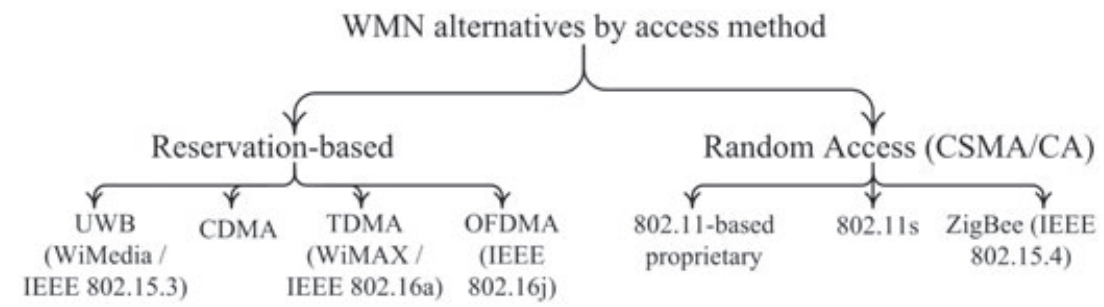

Figure 4. Alternatives wireless mesh networks (WMNs) by medium access control method. UWB, ultra wideband; CDMA, code division multiple access; TDMA, time division multiple access; WiMAX, Worldwide Interoperability for Microwave Access; OFDMA, orthogonal frequency-division multiple access, CSMA/CA, carrier sense multiple access/collision avoidance.

of TDMA-based WMNs. Ultra wideband (UWB) is developed by WiMedia based on IEEE 802.15.3 for WPANs. IEEE 802.15.3 does not, in fact, contain a mesh networking specification, and thus IEEE 802.15.5 Task Group is charged to augment UWB for WPAN mesh networks.

The very definition of the WMNs seems to rule out the intrinsically centralized TDMA and CDMA approaches [6]. The relative scarcity of inexpensive, consumer grade WiMAX equipment prices it out of the reach of many researchers, especially the community and grassroots organizations. In addition, even though WiMAX forum has established three spectrum profiles at 2.3, 2.5, and $3.5 \mathrm{GHz}$, there is no universally agreed spectrum available for WiMAX. It looks highly likely that WiMAX will be deployed over licensed spectrum, and interference issues will be a challenge for researchers to overcome. Finally, synchronization issues of TDMA and code management overhead of CDMA appear to be other major predicaments. Thus, other than very limited and specific-purpose testbeds [38-40], no general CDMA-based WMN has been reported in the literature to the best of our knowledge.

Carrier sense multiple access/collision avoidance-based WMNs appear to be more promising to dominate the future of WMNs because of their distributed method of access, availability, familiarity with large installed base for the underlying 802.11 standard, and low cost. CSMA/CA-based WMNs do have some intrinsic drawbacks, mainly the lack of mechanisms to support QoS ${ }^{\mathbb{T}}$. However, 802.11-based products have experienced a wide market penetration, and all the indications point to a similar trend into at least the foreseeable future. Thus, the rest of the paper will focus on 802.11-based WMNs as the testbeds in the literature are also dominated by them.

\subsection{11 standardization}

IEEE 802.11s [41-46] is a mesh architecture that is an extension to the original basic service set (BSS) for access points (APs) in wireless LANs. The new service set is called mesh BSS (MBSS) and can combine multiple BSSs. The served clients are named as stations (STAs). Each router in $802.11 \mathrm{~s}$ standard is referred to as mesh STA. If this mesh STA also provides access to the Internet or other non-802.11 based networks, it is referred to as portal. A mesh STA can also serve as an AP for the STAs. A sample topology for 802.11s architecture based on the Draft 4.0 [47] (as of December 2009 ) is shown in Figure $5{ }^{\prime \prime}$.

The IEEE 802.11s Draft 4.0 standard uses the Hybrid Wireless Mesh Protocol (HWMP) [46] for routing. The default link metric used in routing is called airtime metric that accounts for data rate, overhead, and frame error rate of a test frame of size $1 \mathrm{kB}$. HWMP utilizes two routing ideas.

\footnotetext{
"The choices for WMNs remind one of the LAN technology front-runners of the early 1990s when Ethernet with its random access method and simplicity had won over the elegant but complicated reservation-based 802.5 token ring protocol.

"802.11s Working Group has been making some changes to the original draft very frequently recently. The newest draft is version 8 published in December 2010.
} 


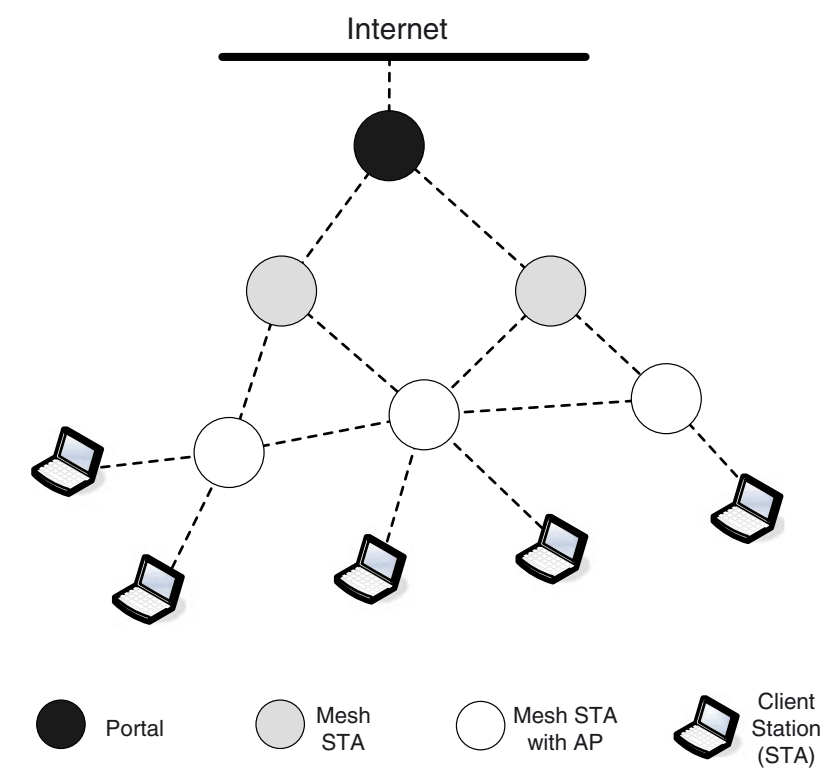

Figure 5. 802.11s architecture. STA, station; AP, access point.

(1) Mandatory proactive tree-based routing that requires a mesh station to be configured as a root mesh station. This root mesh station establishes and maintains paths to all mesh stations in the network.

(2) Once the paths to the root is established, mesh STAs may use ad hoc on-demand distance vector routing (AODV) [48] as part of HWMP to determine shorter paths to the destinations without going through the root. In addition, AODV can be used by itself when no root exists.

Note that because IEEE $802.11 \mathrm{~s}$ has not been ratified yet, the majority of the existing WMN testbeds utilize the routing protocols such as AODV, dynamic source routing [49], and optimized link state routing (OLSR)[50] from MANETs.

\section{WIRELESS MESH NETWORK TESTBEDS AND THEIR TAXONOMY}

\subsection{Wireless mesh network testbed design choices}

Transforming the conceptual vision of WMNs into a more tangible solution depends on many performance and design criteria. In the past few years, several WMN testbeds have been created to investigate their performance under realistic scenarios. Although most of these testbeds are created in academic environments for experimental purposes, there are also several deployments of WMNs to serve a specific purpose and provide Internet access to the public. Whether it be an academic testbed or a deployment, we identified the following design choices to describe the characteristics of the existing WMN testbeds.

3.1.1. Size. This metric indicates the number of mesh routers used in the testbed. Although the size of the academic testbeds can be from small (less than 10 nodes) to large (over 30), for many of the real-world deployments, the size is not reported in publicly available documents.

3.1.2. Location. The testbed can be either indoor, outdoor, or a combination of both. Typically, the academic testbeds are indoors as they are created in university buildings. Real-world deployments are mostly outside as they serve a specific purpose for their users.

3.1.3. Physical/medium access control protocol. The underlying physical and MAC protocols are based on a version of an IEEE 802.11 standard. IEEE 802.11a, b, g or $\mathrm{n}$ can be used. Almost all of 
the testbeds use IEEE 802.11a-based, b-based, and/or g-based routers. With the recent ratification of the 802.11n standard, some efforts have started towards its involvement in testbeds. It is likely that future testbeds will be based on the upcoming 802.11 standardization efforts from the same genre of protocols such as IEEE 802.11ac, 802.11ad, 802.11ah, and/or 802.11af.

3.1.4. Routing protocol. The routing protocol that mesh routers employ to make dynamic routing decisions among the mesh network may vary. The IEEE 802.11s standard, which will designate a standardized routing protocol, is still in development. In the meantime, the protocols from the MANET domain, such as AODV and OLSR, are being used for routing purposes. A classical taxonomy of MANET routing protocols is given in Figure 6. There also exist multiple metrics used by routing protocols to assist in evaluating and selecting the preferred routes between a source and destination on a WMN [51-55]. Traditional metrics, such as hop count, may not provide the performance and reliability desired by WMNs. Researchers have developed and integrated new metrics into WMN routing protocols. These include expected transmission count [56], weighted cumulative expected transmission time [57] and metric of interference and channel switching (MIC) [58]. For a detailed discussion of the WMN metrics, see [51-55].

3.1.5. Hardware type. One unique characteristic of WMNs that has helped the creation of many testbeds is the availability of inexpensive hardware platforms that can allow modification and running of open-source software. There are several options that can be pursued to build the routers in WMN testbeds under different software platforms. Figure 7 shows the alternatives for the WMN node hardware. By far, the most common type of WMN router is the one custom built using small, single-board x86-based PCs. These devices typically utilize a small compact flash card or other type of memory for operating system installation and have wired Ethernet interfaces and mini-peripheral component interconnect (PCI) slots for the installation of various types of wireless network interface cards (NICs). The single-board PCs are installed in custom cases and are equivalent in size to many of the home WiFi routers sold at electronics and discount stores. The advantage of these nodes is that they have a much more open architecture than WiFi home routers. One can adjust hardware components, such as changing the type of wireless card from $802.11 \mathrm{~b}$ to WiMAX in order to test them separately.

In addition to custom-built routers, various home routers have also been used as mesh routers. The low price, availability, and quasi-open nature of wireless home routers have made them an attractive platform for WMN routers. There are several open-source firmware replacements that, when installed, provide a wealth of additional features and functionality to these home routers. The most common firmware used by WMN researchers is OpenWrt [59]. Several research groups have

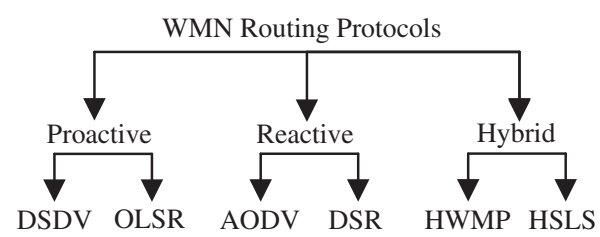

Figure 6. Classification of mobile ad hoc network routing protocols used in wireless mesh network (WMN). DSDV, destination-sequenced distance-vector routing; OLSR, optimized link state routing; AODV, ad hoc on-demand distance vector routing; DSR, dynamic source routing; HWMP, Hybrid Wireless Mesh Protocol; HSLS, Hazy-Sighted Link State Routing Protocol.

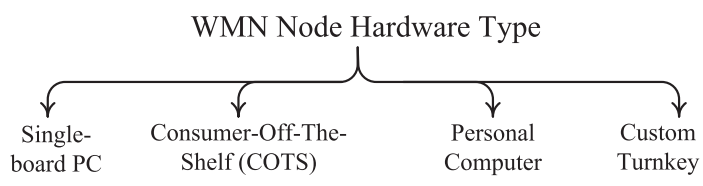

Figure 7. Wireless mesh network node hardware alternatives. 
built their own custom distributions providing WMN networking from the OpenWrt platform and released them for public use.

A third class of hardware routers is based on full-sized or small-form factor x86-based PCs. Although many may have been repurposed, these types of machines are the most costly platform, requiring the largest physical footprint and power resources. Conversely, they also offer the highest performance, flexibility of radio types, and operating systems. The size of these devices allows them to accept full-sized PCI expansion cards as well as mini-PCI cards with the use of special adapters. This fact increases the range of available hardware radio devices that can be used for the WMN testbeds. Further, the choice of operating systems that can be installed for the WMN router is enormous, with thousands of open-source distributions as well as commercial offerings available to choose from.

Finally, there are some turnkey (ready-to-use) boxes available that are customized for operating as WMN nodes. They come with all the hardware and software needed to operate as WMN nodes with some configuration options available through the software interface. Examples include Meraki (Meraki Inc., San Francisco, CA, USA)[60], Tropos [25], Open-Mesh [26], LocustWorld (LocustWorld Ltd, Surrey, UK) [61], and so on.

3.1.6. Software platform. This is the operating system used for running the WMN protocols. The choices are wide ranging. Although it is required that routers support nearly every available operating system for client devices connecting to them, the overwhelming choice for router operating systems is some UNIX variant, such as OpenWrt [59], Ubuntu, and NetBSD. Many research testbeds take common distributions and make enhancements and modifications to them, whereas others create their own from the bare-bones Linux kernel. The flexibility and open nature of Linux allows for an array of implementation options.

Although Linux is certainly the dominant operating system, it is not the only one. Microsoft at one time had a research department dedicated to wireless mesh networking and even released their software for public. Their Mesh Connectivity Layer (MCL) [62] is a Windows loadable driver that provides self-organizing mesh connectivity for modern Windows operating systems. They did release an academic toolkit and source code for the MCL that has been used in at least two academic testbeds $[63,64]$. The MCL project now appears to be defunct.

3.1.7. Use of channel assignment. The strategy used for the channel assignment in mesh routers may vary. Despite the advantages of the multi-NIC, multiradio, multi-antenna and/or multichannel configurations in terms of increased network span, improved scalability, more robust dependability, and higher throughput, initially WMNs have been mostly built with a single radio and a single channel.

In a multichannel, multiradio, multi-antenna and/or multi-NIC WMN, a crucial component is the channel assignment strategy, which is a nondeterministic polynomial-time hard problem [65]. The channel assignment problem involves obtaining an efficient mapping of the available radios to channels with the goal of minimizing interference among routers in the WMN to effectively utilize the available bandwidth. Whereas most of the literature considers channel assignment problem in isolation of other research topics, some of them study channel assignment jointly with routing.

The channel assignment approaches for WMNs can be categorized based on how (i.e., dynamic or static) and where (i.e., centralized or distributed) the assignment is performed. Figure 8 shows a simplified taxonomy of WMN channel assignment techniques. Whereas in static channel assignment

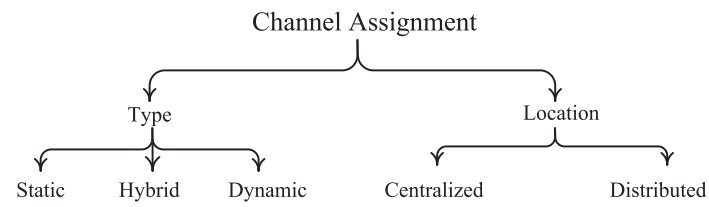

Figure 8. Categories of channel assignment approaches. 
[65-73] all the nodes are assigned to their channels at the beginning and do not change their channels throughout their operations, dynamic channel assignment [74-77] can change the assigned channels after the initial channel selection. That is, a node may switch to another channel based on the traffic and interference changes during data transmissions. In the hybrid channel assignment [78-81], some of the channels are fixed and the rest are dynamic. In this way, the initial topology can be preserved and at the same time the nodes can adapt to changes in their neighborhood easily. An overwhelming majority of the assignment techniques use static approach as dynamic channel switching has a delay overhead.

Another type of categorization for channel assignment is based on where the decision to change the channel assignment is done. Specifically, there are centralized and distributed approaches. In centralized channel assignment, the assignment is done at a node that knows the whole network topology. This is not the case in distributed approaches where the assignment is performed locally among the nodes. Thus, they can scale better and the cost of changes to the assignments can be handled locally.

3.1.8. Wireless configuration options and radio. A WMN node can be deployed with a variety of different radio and channel switching configurations options. Channel switching is the ability of a node to move from one radio channel to another, such as from $802.11 \mathrm{~b}$ channel 1 to channel 6 when interference on channel 1 is high. Those that do not employ channel switching use a static channel assignment method. A node may contain a single radio interface or a combination of multiple identical or dissimilar radio interfaces.

Some testbeds using multiple radios contain dual $802.11 \mathrm{~b} / \mathrm{g}$ cards, for example, whereas others may contain one $802.11 \mathrm{~b} / \mathrm{g}$ radio and a secondary $802.11 \mathrm{a}$ radio. The antenna type is also a concern of WMN testbed designers. Antennas affect the propagation of radio waves in conjunction with the propagation environment and the quality of the signal itself. The use of long-distance directional antennas as compared with omnidirectional antennas will change the coverage area and WMN testbed considerably. Finally, the number of antennas can be adjusted. For example, MIMO technology can use two or more antennas. Conceptually, a WMN node will have many wireless configuration options as depicted in Figure 9 for the WMN testbed designer to choose from.

\subsection{Taxonomy of wireless mesh network testbed design choices}

A comprehensive classification of the existing WMN testbeds is provided in Table I in terms of the testbed design choices as outlined in Section 3.1. Note that because many of the testbed descriptions lack a detailed discussion of wireless options as given in Figure 9, we have included a coarser classification in Table I under Radio column only with respect to number of radio interfaces available. In addition, under the Channel Assignment column, we have only included what we can deduce from the published testbed descriptions.

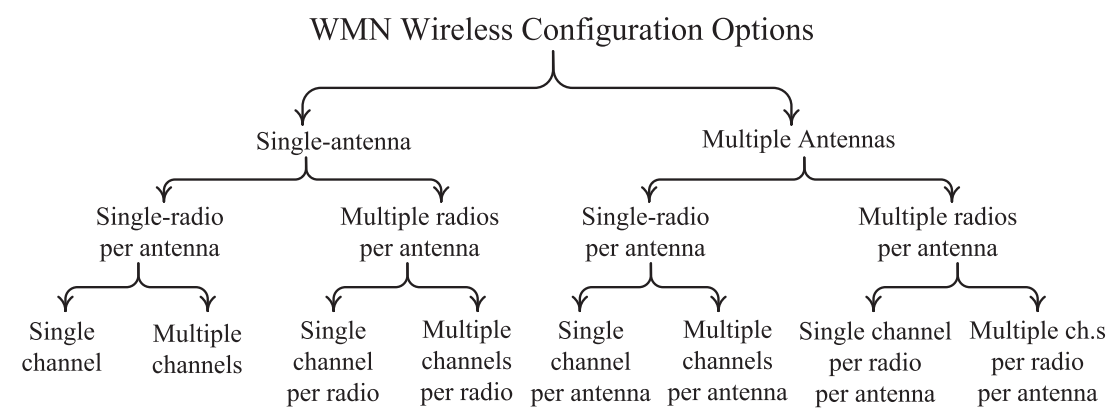

Figure 9. Wireless mesh network (WMN) node wireless configuration options. 


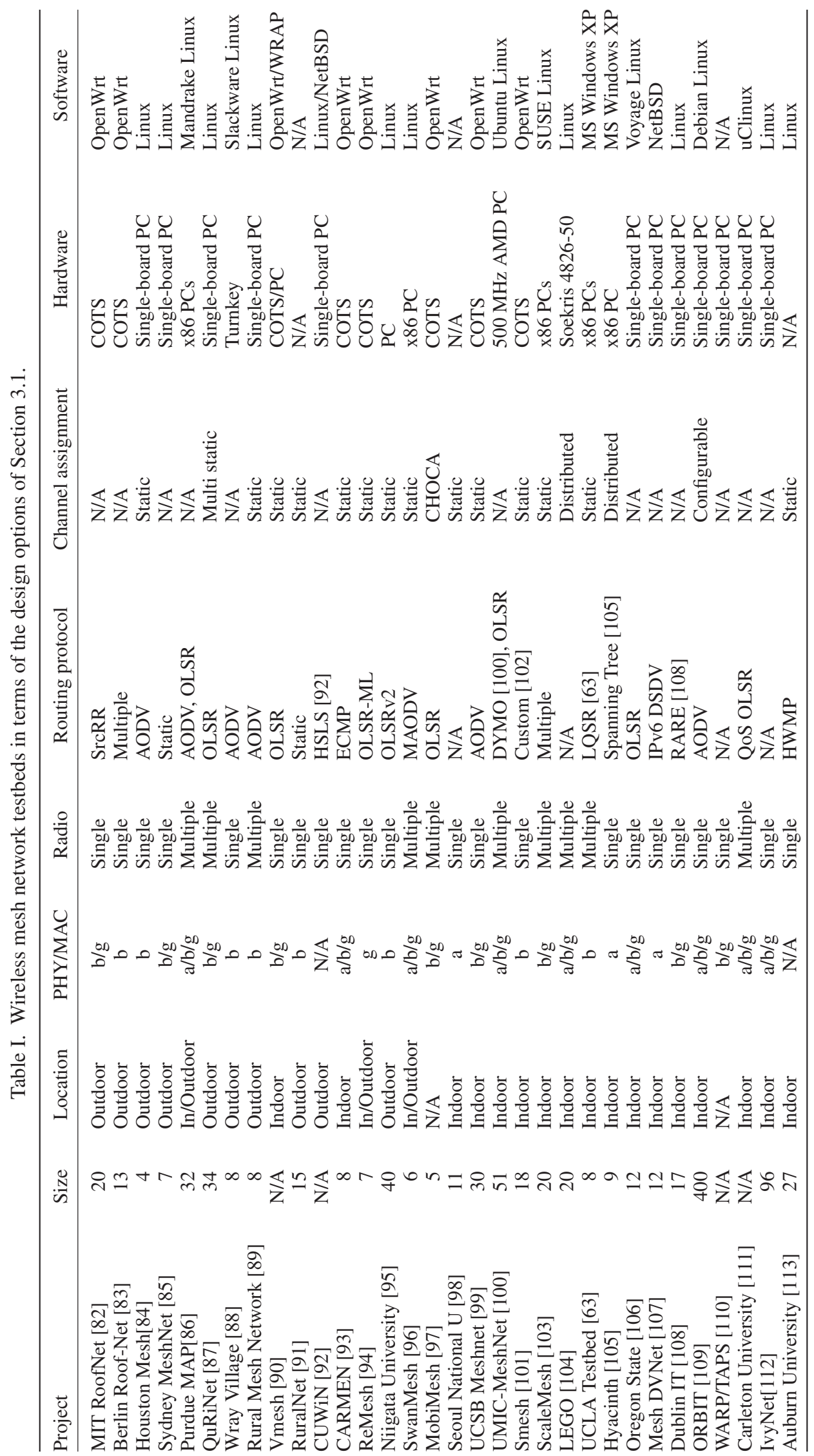




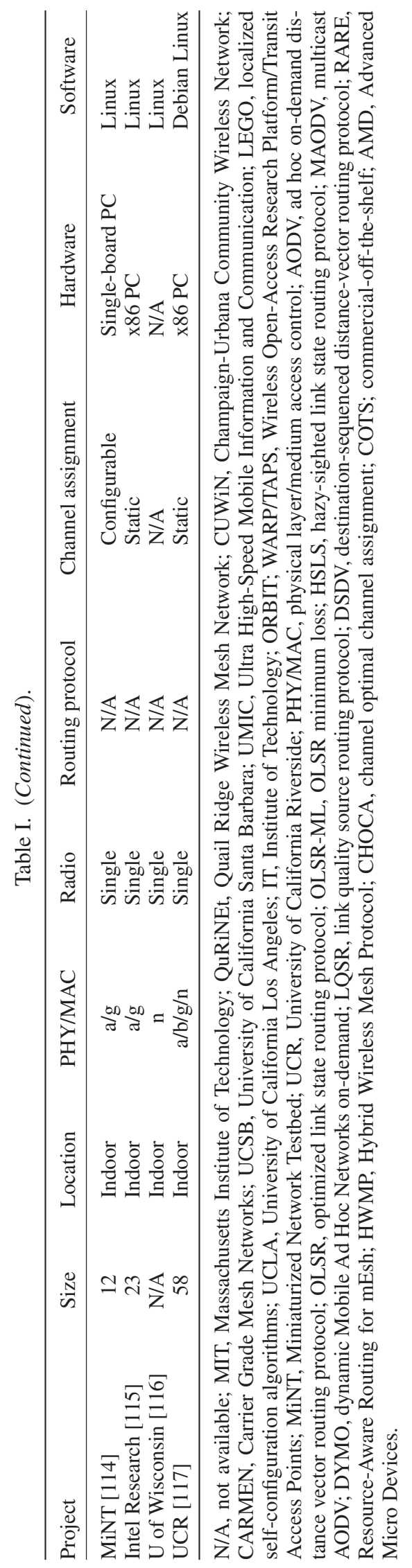




\subsection{Research goal-oriented wireless mesh network testbed taxonomy}

Given the testbeds in Table I, we have categorized them based on their research goals as seen in Figure 10. These research goals are broadly in four categories: (i) anything related to routing; (ii) mobility of the nodes and clients; (iii) any issue regarding interference or throughput improvement; and (iv) issues regarding QoS at different layers. Note that most of the testbeds with a real-life deployment serve for broadband Internet access and may not have any major research goal. Therefore, some of the testbeds may not be listed in the proposed category if they do not have any research goals. In addition, some testbeds test multiple issues from different layers of the protocol stack. As a result, a testbed may be categorized in multiple research categories.

This categorization indicates that the majority of the testbeds has focused on routing-related research experiments such as testing the performance of new routing protocols, new routing metrics, IPv6, and multicast routing. Another widely focused research category is related to interference and thus improving throughput in the network. This is not surprising as interference-related research may not be realistically performed with simulators. Detailed treatment and discussion of the impact of imprecise interference settings with respect to physical realities can be found in [15-17, 19-21,23]. Other issues such as QoS and mobility have also attracted experimentation in various WMN testbeds.

\subsection{Discussions of testbed choices for research goals}

To avoid the potential future roadblocks in experiments and to reduce the likelihood of inaccuracies or outright ambiguities in testbed outcomes, it is wise to make the testbed design under explicit consideration of the expected research goals. In other words, a WMN testbed initiative should begin with a somewhat clear picture of the ultimate research or deployment goals in mind, along the lines of the rather coarser granularity classification given in Section 3.3. Drilling down to finer, more specific goals may also be articulated if such information is known in advance. The testbed design choices, as detailed in Section 3.1, should be decided with the aforementioned goals in mind to obtain the best benefits. For example, scalability and robustness or providing satisfactory performance under failures in the network are two major sources for routing algorithms in WMNs. If the research goal is to evaluate a new routing protocol, then the testbed should be able to accommodate a reasonably large number of nodes, and the testbed design should allow for many routing protocols and metrics to be run in order to compare and evaluate the new routing approach with the existing

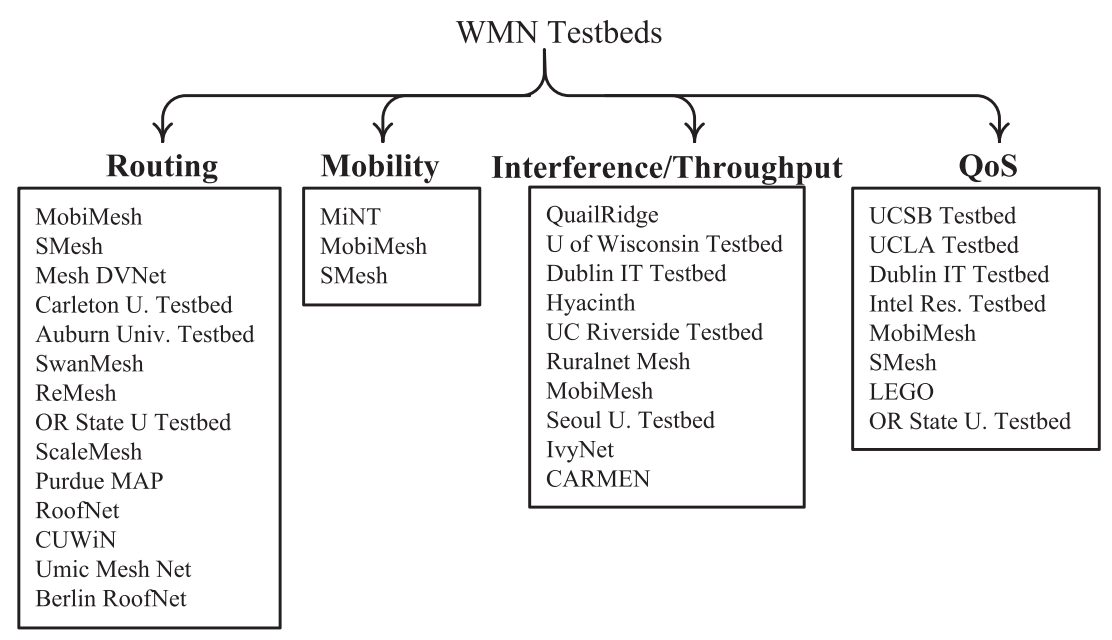

Figure 10. Wireless mesh network (WMN) testbed taxonomy based on four primary research focuses: routing, mobility, interference, and QoS. See Table I for references of the testbeds. CUWiN, Champaign-Urbana Community Wireless Network; IT, Institute of Technology; UC, University of California; CARMEN, Carrier Grade Mesh Networks; UCSB, University of California Santa Barbara; UCLA, University of California Los Angeles; LEGO, localized self-configuration algorithms. 
ones in meaningful ways. Another use case of the presentation of this section by testbed developers may be given when the research goal of the testbed project is to assess a new channel assignment mechanism. With that ultimate goal in mind, the designers may depict a clearer road map for developing the testbed by considering the taxonomies provided in Figures 8 and 9 for the alternates. For example, the number of channels per radio per antenna within the framework of Figure 9 should be decided from the onset to avoid unnecessary repetitions and jeopardizing the scope of the applicability of the results. More operational level examples can also be given, but we would like to stay at a rather conceptual level to reduce the length of the paper.

\section{EVALUATION OF WIRELESS MESH NETWORK TESTBEDS}

One of the primary motivations for this study is to explicitly define a set of desired features for WMN testbeds and to determine which of the features the testbeds surveyed have been successfully implemented or attempted to be implemented. The list of testbeds surveyed is quite expansive, and not all identified desirable features are explicitly discussed in literature related to each individual testbed. Therefore, we provide an analysis of successful feature implementations based on the publicly available literature associated with each testbed. Then, A discussion of the applicability of these features for different testbeds follows. This section concludes with an evaluation of the existing testbeds using these features as shown in Table II.

\subsection{Desirable wireless mesh network characteristics}

We first discuss the desirable features/characteristics of future WMN testbeds. We would like to note that this list is not exhaustive and does not necessarily indicate that all the features are desirable for every testbed or deployment. We will discuss some high-level, conceptual use cases for desirable features of testbeds in Section 4.2. Depending on the application, the set of desirable features may vary, and this can be identified by the testbed designers for the particular set of goals they are seeking.

4.1.1. Lower cost. An important cost category of testbeds is hardware. Development, setup, and maintenance costs can be significant too, although they are much more difficult to quantify and so are omitted from this particular discussion. As mentioned in Section 3.1.5 and shown in Figure 7, testbeds surveyed are implemented on four main platforms: small office/home office routers (low cost), also called as commercial-off-the-shelf, custom single-board PCs (medium cost), full-size desktop computers (high cost), and custom, turnkey black boxes. The small office/home office routers and other commercial-off-the-shelf hardware are typically the most cost-effective but are not quite as flexible for node deployment as those based on PC platforms. Additional components, such as secondary wireless radios and antennas, increase hardware cost. Nearly all testbeds are implemented on freely available software platforms such as OpenWrt, FreeBSD, or a Linux variant, so software cost is typically negligible. Turnkey nodes may provide the most convenience at the expense of diminished flexibility of feature set.

4.1.2. Network management. There are two features that can be considered under the 'Management' umbrella:

(1) Network monitoring/administration: The initial deployment of testbed nodes and the subsequent monitoring and maintenance are key components to building a testbed encompassing desirable features. Nodes should ideally be relatively easy to configure and deploy and have an efficient method of connecting for reconfiguration, restoration, troubleshooting, and software updates. Because nodes might be located in areas difficult to access, such as roofs, light poles, and volunteer's homes, reliable access for management is critical. Testbeds can implement management through either in-band or out-of-band signaling. The latter is preferred as it should have minimal effect on the experimental radio interfaces. Secondly, the current operational status of the nodes and the entire network should be available quickly and easily. Finally, it is essential that data collection from individual nodes be reliable, and automatic collection 


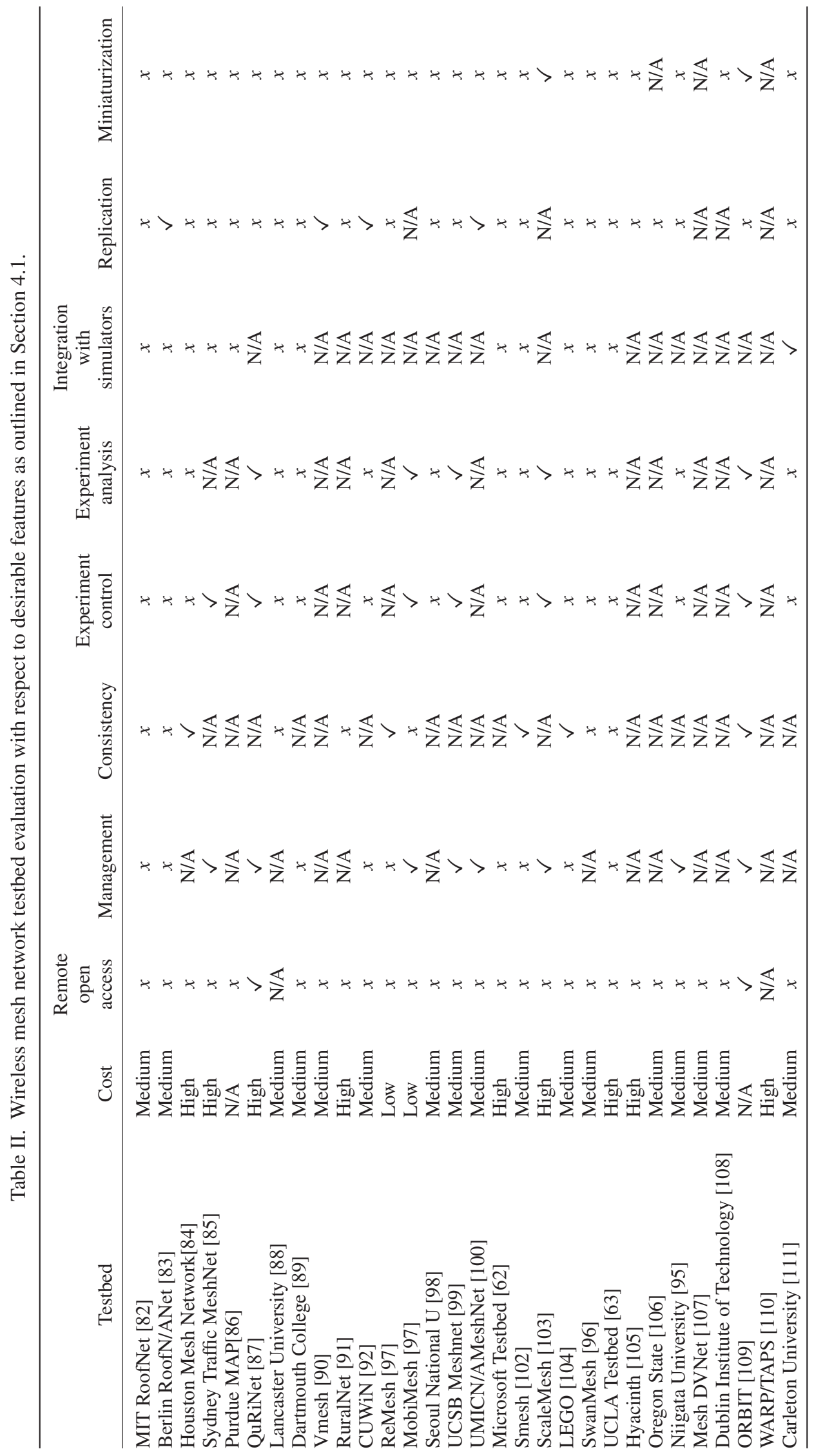




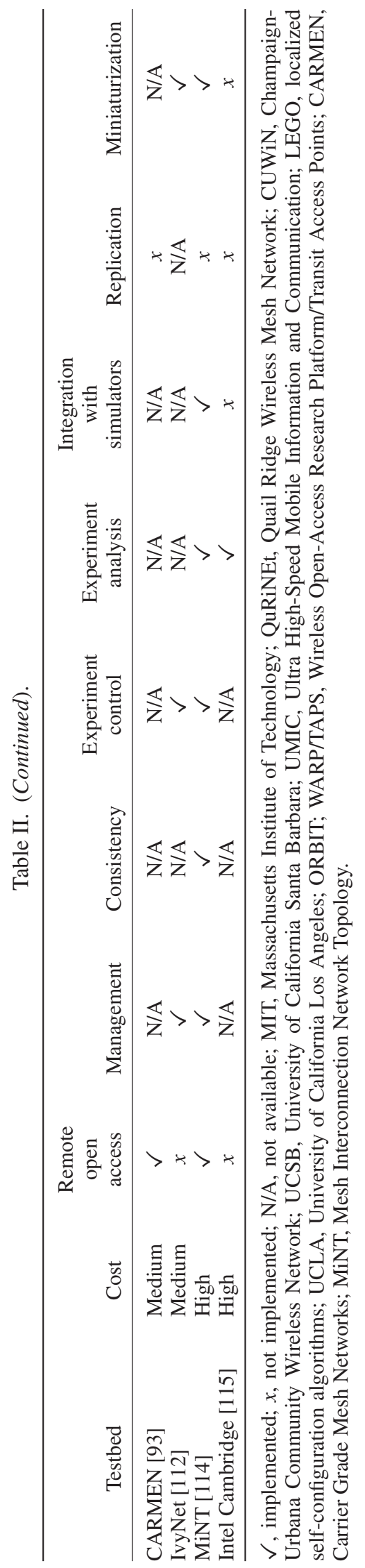


is preferred. Although the problem of network monitoring has been well studied in wired networks (i.e., Simple Network Management Protocol (SNMP)), the issue becomes complicated in wireless networks and even more so in multihop WMNs. First, there is no single, widely adapted network management standard for wireless networks unlike the ubiquity of SNMP for wired networks. Second, the management becomes quite complicated for multihop WMNs $[118,119]$ because of hierarchical network topology, connectivity maintenance, device mobility, and routing challenges. Thus, the usefulness of SNMP is greatly curtailed for WMNs. Third, the distributed and self-organizing nature of WMNs render SNMP less relevant as SNMP was developed for centralized paradigms [120]. Fourth, the overhead of SNMP in WMNs in bandwidth is very high $[121,122]$. Fifth, network layer connectivity issues make SNMP in WMNs ineffectual [121]. Finally, SNMP fails to retrieve all interesting and relevant statistics, such as the currently used node or number of hops to it, in WMNs [122]. Therefore, the testbeds providing this feature implement their own methods as there is no standardization yet.

(2) Remote open access: Under the management feature, an important design option is remote open accessibility. In fact, a recent trend in networking research is the creation of 'open' testbeds that allow for researchers outside of the testbed owner's institution to access the testbed for the purpose of experimentation. This access could be either physical (on-site) or more naturally through remote connectivity. The latter being preferred because of the greatly expanded hours of availability and subsequent decrease in manpower expense to hosting institutions. Remote access, via secured virtual private network, and Internet connectivity, could open the testbed for 24/7 access for researchers across the world, allowing for greater collaborative opportunities and access to those with little funding or equipment but with strong interest in WMN research. In this respect, the testbed should have an easy-to-use scheduling system to prevent conflicts. Obviously, this feature may not apply to real-life deployments that are already open to public for use.

4.1.3. Experimental issues. There are three desirable features regarding the experiments as explained further:

(1) Consistency and repeatability: The very nature of wireless communications makes, for achieving total consistency across multiple trials of the same experiments, an impossible task. Physical phenomena, such as interference, attenuation, and fading, are a real concern for wireless testbeds. Controlling these as much as possible while still delivering an accurate, real-world environment is a tough task for testbed designers but still a goal worth striving for. This feature would be particularly valuable for the testing of a single parameter on the performance of the overall WMN without being affected by the varying environmental factors. Academic testbeds are more suitable to provide this feature as real-life deployments will be subject to varying effects from the surroundings and human beings. However, realistic wireless behavior and performance should not be compromised in order to attain a higher level of experimental consistency. Therefore, having full control over adjusting the effects of the environments is desirable. A wireless emulator reported in [123] may be a good choice for WMN testbed to increase the chances of experiment repeatability. Another approach for consistency and repeatability that uses electronically controlled wireless channels for repeatable wireless environments, such as different levels of path loss, multipath fading, and interference, is explained in [124]. A topological repeatability tool developed in [125] for wireless networks may also provide a satisfactory approach for WMN testbeds in terms of repeatability.

(2) Experimental control: There are several concerns and requirements in creating a WMN testbed that allows for the necessary amount of control for experiments. The authors of [126] have identified six components of experimental control that contribute to the overall success of designing an experiment on a WMN testbed. Further, conducting experiments in places where uncontrollable wireless environments (called RF Jungles as coined by the authors in [127]) may lead to misrepresentations. The topology has a tremendous effect on experiments, as distance, physical impediments or obstructions, and even atmospheric conditions can cause 
variations in wireless communications. A testbed that can provide multiple alternatives of simulated or real application data to send across the network would be ideal. For researchers seeking to study the mobility effects, the ability to control nodal movement is desirable. Researchers may find it beneficial to modify or tweak configuration parameters for general platform or specific radio settings. These modifications should allow for the user to save them and reuse them as necessary and still allow for quick restoration to defaults for other users. Experimental execution flexibility, including the starting/stopping/pausing/restarting of experiments, making adjustments as needed, and general ease of initiating experiments, are also desired. Finally, protocol-debugging ability, in order to identify protocol problems or misconfigurations, is essential. Such a feature would reduce the testing time significantly as bugs can be quickly identified. In this regard, one possible option is to be able to visualize the behavior in the network by using specially developed visualization tools.

(3) Experiment analysis: The main purpose of a testbed is to conduct research experiments on new technologies or using new methods or protocols on established technologies. In this regard, ensuring reliable and accurate collection of experimental data is critical in justifying the value and contribution of a testbed and its validity. The results of the testbed experiments are arguably the most vital component associated with a research project. One common data type for network testbed experiments is trace data. Network trace data should be easy to receive and review, using a standard format, such as that used by tcpdump [128] or Wireshark [129]. The traces should be easily aggregated with an interface to allow visual examination of the trace data for specific information. Filters should also be available for narrowing down trace data to only what is relevant for a given experiment. This is also closely related to the debugging feature discussed under 'experimental control' because such trace data would facilitate debugging efforts greatly.

4.1.4. Integration with simulators. An ideal testbed will offer the experimenter access to multiple physical layer technologies. This survey has focused on 802.11-based testbeds that have used $802.11 \mathrm{a} / \mathrm{b} / \mathrm{g} / \mathrm{n}$ standards. Connectivity for $3 \mathrm{G}, 802.16 \mathrm{e}$, ZigBEE, or WSNs provide an added benefit. As shown in Figure 11, we are facing an ever-increasing heterogeneity in wireless networks. As the dissimilarity of wireless networks proliferate, the practicality of developing all-encompassing WMN testbeds is becoming more difficult. Thus, to accommodate the heterogeneity as depicted in

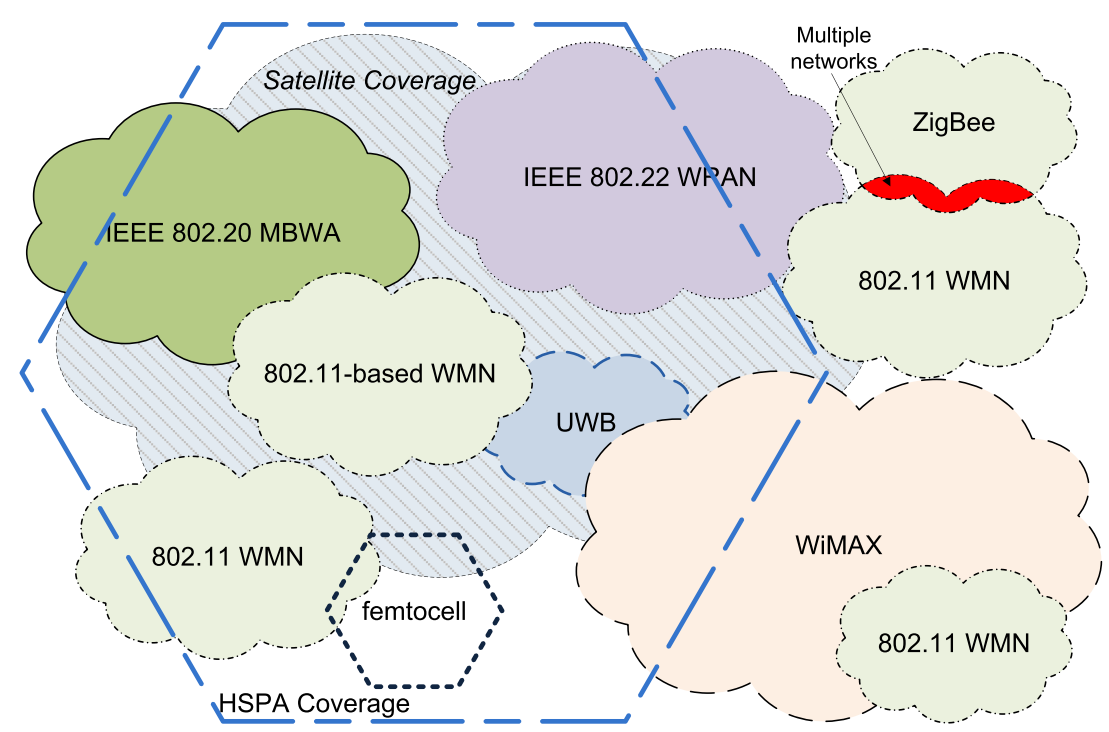

Figure 11. Heterogeneity in wireless communications. MBWA, ; WPAN, wireless personal area network; WMN, wireless mesh network; UWB, ultra wideband; WiMAX, Worldwide Interoperability for Microwave Access; HSPA, High-Speed Packet Access. 
Figure 11, the ability to integrate simulations with the testbeds emerges as another crucial feature for the testbeds of the future. A hybrid simulation environment, where portions of the network layers are run in simulation, whereas others are not, is considered to be highly desirable. Using real hardware for physical and MAC layer activities and simulators for network, transport, and application layers is an example of a hybrid simulation. Such an integration with simulators may provide opportunities to test various mesh topologies and node mobility that are typically hard to test in a real testbed. It is obvious that many other useful previously infeasible performance evaluation scenarios are likely to unfold as this features becomes more common.

4.1.5. Replication. The ideal testbed should be easy to reconstruct by other researchers and institutions. Literature and documentation should provide detailed instructions on implementing the WMN testbed. Software and hardware should be widely available and universally supported. Another important aspect with regards to convenient replication is the support of pre-deployment testing. If the testbed designers provide tools/software to test the protocols on the hardware to be used before going to the deployment, this would significantly decrease the deployment time frame. Although this is not something directly related to the testbeds, it should be considered as part of the testbed creation efforts, particularly for academic testbeds.

4.1.6. Miniaturization. A final desired feature is miniaturization. The idea is to develop a testbed in a smaller physical space without sacrificing accuracy [130]. Miniaturization offers real-world testing scenarios by means of smaller nodes, lower transmit and receive power, and smaller physical site area by mimicking the settings of the full-blown environment at a reduced scale. Although this might not be ideal for all WMN experimentation, this feature is certainly one that can provide researchers many benefits. In this way, several features regarding scalability can be tested in a laboratory environment without deploying the nodes inside the buildings or outdoors. Obviously, this feature is not an option for real-life deployments.

\subsection{Discussion on applicability of desirable testbed features}

Our goal in the presentation of desirable testbed features earlier has not been meant to put them forward as a one-size-fits-all type of shopping list for all testbeds. As we have stated in Section 4.1, we do not envision to have all those desirable features equally applicable to all kinds of WMN testbeds. Further, some of the features may even be irrelevant or completely undesirable for some testbeds. Remote open access feature is likely to be important and desirable for most, if not all, research testbeds. On the other hand, it may very well be not desirable for some testbeds as stated earlier. Remote open access may not be necessary for some esoteric security concerns, or even undesirable for community testbeds. In a nutshell, we expect lower cost, network monitoring, ease of maintenance, consistency, repeatability, precise experimental control capability, reliable and accurate collection of trace data, and ease of replication to be indispensable aspects of all the testbeds. Operational level constraints may, however, prevent these characteristics to be a part of every testbed, of course. However, lack of these aspects simply could not negate their potential added value were they to be available. Being a desirable feature is one thing, whereas lack of it in a particular testbed because of some resource constraints is another thing.

Logistical restrictions may make miniaturization of the testbeds a requisite property, especially for those performance evaluation cases where scalability is of an important concern, such as routing protocol evaluation, channel assignment assessment, and so on. Finally, integration with simulators would considerably extend the scope of the performance evaluation domain to those technologies or equipment whose inclusion in the physical testbed is not feasible for monetary, practicality, or other reasons. For example, if a new network routing algorithm is to be tested under different MAC protocols, it would not be possible to test very recent MAC standards or those under development. More specifically, it would be impossible to find any equipment conforming to the upcoming IEEE 802.11ac (next-generation Gigabit WiFi), for instance, in a physical testbed for quite a while. However, once a draft standard is released and a simulation is developed, then at least some results can be obtained in a testbed for new ideas using the 802.11ac standard in conjunction with the existing 
technology. This should make transitioning to the new technology easier to plan and test at the very least, besides other potential benefits.

\subsection{Taxonomy of wireless mesh networking testbeds in terms of desirable features}

A summary of classification for the existing testbeds within the framework of the features outlined in Section 4.1 is given in Table II. As indicated earlier, not all of the features studied in Table II will be relevant for all the testbeds; it just provides a collection of desirable and significant features WMN testbeds of the future will likely be pursuing.

\section{OPEN RESEARCH ISSUES}

In this section, we first list the open future issues in the design and development of WMN testbeds. Then, we turn our attention to the research issues to be tested in WMN testbeds, as the majority of the testbeds surveyed for this paper are used primarily for research purposes. Although much research has been conducted on WMNs using the surveyed testbeds, there are a variety of open issues that must still be examined in order for the technology to progress further. We categorize and describe some of the open issues, waiting to have their performance evaluated in realistic settings with respect to their protocol layers. We would like to note that although some of these issues may have been already listed in [6] as WMN future research, our focus will be from the testbed perspective and thus point out why testbed development and testings are essential for such future research issues.

\subsection{Open Issues on wireless mesh network testbed development}

We have identified the following open issues for the development of future WMN testbeds:

(1) Network monitoring: Future WMN testbeds should provide network monitoring as an essential part of the academic as well as real-life testbeds. Standard protocols, similar to the wide use of SNMP in wired networks, should be developed. However, such a standard should consider the characteristics of WMNs (i.e., wireless environments, security, and low bandwidth) in order to be realized as articulated in Section 1 with respect to deficiencies of SNMP for WMN testbeds.

(2) Debugging tools: Given the lack of debugging tools in the current WMN testbeds, this is one of the most important open issues that need to be addressed for future WMN testbeds in order to minimize the deployment and testing times. This may also include a visualization tool that provides visual representation of data flows among the nodes in the WMN.

(3) Integration with simulators: To support various type of experiments under different topology and mobility patterns and still providing the realistic nature of wireless environments, such a support is very much needed in future WMN testbeds. Currently, almost no WMN testbeds fully support this feature.

(4) Support of multiple radios with various MAC mechanisms: The nodes in future WMNs are expected to have multiple radio interfaces that can support different MAC technologies such as WiMAX, 3G, or Zigbee. Therefore, the future testbeds should offer such options in order to be able to experiment with these emerging technologies.

(5) Support for pre-deployment testing: As the testbed deployment is a cumbersome task where developer may face unexpected hardware and software problems, it will be crucial to support pre-deployment testing for the WMN nodes. That is, the proposed protocols can be tested with a software tool that emulates the hardware characteristics of the WMN nodes. These type of tools have been used in WSNs for Mica Motes (Crossbow Technologies Inc., Milpitas, CA, USA) [131]. TOSSIM [132], for instance, is used to emulate the behavior of Mica Motes before the real prototype is deployed.

\subsection{Open issues to be testbed on wireless mesh network testbeds}

The following issues at different protocol layers are identified as future research issues to be tested on WMN testbeds. What follows provide a snapshot of the major issues due to the ever evolving 
and dynamic nature of the field. Yet, we believe that the researchers working on WMNs can benefit from this list.

5.2.1. Physical layer. Physical layer technologies tend to exhibit the most promise to aid in increasing data rates and reduce bit-level errors. Performance evaluations of MIMO, co-channel interference, wideband transmission (such as orthogonal frequency division multiplexing, UWB, etc.) on spectrum efficiency, relative efficacy of dynamic channel coding schemes, software-controlled physical layer parameters such as radio channel assignment, directional antenna, and antenna switching in multiple antenna scenarios would benefit significantly from experiments in realistic testbeds. These problems are further compounded in different environments such as underground, underwater, and tunnels. Thus, for some applications, testbeds would be the only way to conduct meaningful experiments.

5.2.2. Medium access control layer. Medium access control layer for wireless devices have been traditionally implemented in hardware or firmware. The recent trend is to move it to software for a more versatile and flexible operation. This shift alone will need significant performance evaluation effort, and testbeds can contribute very meaningfully. MAC schemes have been developed for single-channel and single-hop operation. There is a need for new MAC protocols proposed with explicit multiple channel considerations under multiple hop topology. In addition to this paradigm shift, the MAC layer will need to get involved in choosing the channel, the radio, the antenna and/or the interface card for optimum transmission. Finally, the ability for MAC protocols to integrate properly and efficiently with routers that connect to $802.11,802.16$, ZigBee, WSNs, and other technologies not yet available is an important feature. Testbeds would be instrumental in assessing the different techniques to be proposed in the coming years.

As far as the channel assignment strategies are concerned, testing of dynamic channel assignment schemes in testbeds has drawn little attention so far. One such study is given in [80]. The performance of routing and MAC protocols with respect to dynamic channel assignment schemes needs extensive evaluation before dynamic approaches can be realized.

Standard-related issues also require testbed evaluations. The recently ratified IEEE $802.11 \mathrm{n}$ has introduced many new features, such as channel bonding, beamforming, and so on, whose pros and cons under different scenarios are in need of further detailed studies. In addition, the tests for checking its performance with existing MAC protocols, such as IEEE 802.11a, b, and g, should also be conducted for interoperability and performance under heterogeneous environments. The upcoming IEEE 802.11s as a WMN standard will need to have its performance studied under a variety of conditions. The new wireless standards that are likely to form the infrastructure of the future WMN testbeds will also need reliable and validated testbeds to evaluate their performances. Examples of these kinds of standards under development include IEEE 801.11ac, 802.11ad, 802.11af, and 802.11ah.

5.2.3. Network layer. At the network layer, multihop MANET routing protocols have served as a starting point. We still do not know much about the routing performance under realistic scenarios. WMN features, such as topology changes and inconsistencies, coupled with the inherent difficulties in resource allocations, call for a new genre of routing protocols. A whole set of routing metrics have been proposed for WMN routing, but a comprehensive, representative, and comparative analysis is still lacking. Multicast and QoS routing algorithms and protocols, likely to be forthcoming in the next few years, will need to be evaluated fully in realistic testbeds, too.

5.2.4. Transport layer. Transmission Control Protocol's roundtrip time mechanism and its congestion control technique will need meticulous evaluation for the WMN traffic. The User Datagram Protocol for multimedia traffic needs more work carried out as well in terms of delay, delay jitter, and loss measurements as experienced by applications. Testbeds could provide more relevant and realistic testing tools than simulations. 
5.2.5. Application layer. Application level parameter tuning needs to be tested for QoS applications, such as VoIP and multimedia streaming. In particular, multimedia streaming seems to find emerging applications in community WMNs, where the traffic does not travel through an Internet gateway. Video encoding and compression techniques are yet to be tested for such applications under realistic WMN testbeds.

5.2.6. Multilayer experiments. Cross-layer design seems to be getting more important than ever for WMNs [6]. Cross-layer design involves WMN protocols or applications that are aware of and often can communicate across the traditional boundaries of the communications reference model layers. One example that could be considered is that of a network layer routing protocol that is able to observe and consider wireless link interference, a physical layer property, when making decisions about routes. On the other hand, there are many pitfalls in the cross-layer design [133] and the performance of cross-layer protocols should be examined in testbeds in addition to the current efforts for more effective analyses. Figure 12 provides the most commonly used examples of cross-layer design possibilities.

5.2.7. Others. Security at every layer is a significant challenge that must be considered when designing or deploying a WMN. Very few of the surveyed testbeds have paid particular attention to studying security issues on WMNs. Although traditional wireless networks have security frameworks, such as $802.11 \mathrm{i}$, whether these function adequately on WMNs is yet to be seen. Secure node association and authentication, secure data forwarding, and secure routing and its effects on the performance of QoS routing, are among the topics waiting to be evaluated in testbeds.

Mobility management, network monitoring, and management, and centralized versus distributed approaches are other interesting topics that could benefit from extensive experiments on testbeds.

Finally, with the increasing interest in the outdoor deployment of WMNs in applications such as smart grid, wildlife monitoring, and so on, power-efficient hardware and software are likely to become a concern in the near future. Therefore, testing of power-efficient protocols and hardware on the testbeds will be an important contribution.

\section{CONCLUSION}

In order for the potential and expectation of the WMN concept to materialize, a significant research effort needs to be exerted. In addition, it seems to be underway for the past few years. Although a lot of testbed-based studies have been conducted, still the majority of the performance evaluation of WMN research has been simulation-based. The inherent complexities of WMNs make it very difficult, if not impossible, to model the underlying wireless network infrastructure accurately. With the imprecision in the modeling, it would not be too unrealistic to question the validity of many of the performance evaluations carried out only in the simulations. Thus, testbeds would bring in a significant value to the more proper and realistic experimentation for WMN research. Yet, the testbeds are not at the level where they can start catalyzing the aforementioned benefits for WMN research. With this paper, we have striven to draw attention to the lack of comprehensive testbed infrastructure, even though many excellent, but insufficient, works exist. In addition, we have identified ideal WMN testbed features to analyze the current WMN testbeds. The analyses and discussions can be utilized by researchers to assist in constructing a testbed that can provide the most benefit to its designers and users.

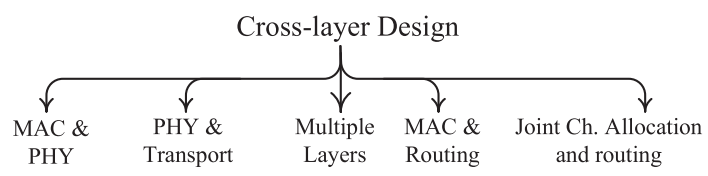

Figure 12. Cross-layer protocol design combinations. MAC, medium access control; PHY, physical layer. 


\section{REFERENCES}

1. Bruno R, Conti M, Gregori E. Mesh networks: commodity multihop ad hoc networks. Communications Magazine, IEEE March 2005; 43(3):123-131. DOI: 10.1109/MCOM.2005.1404606.

2. Akyildiz I, Wang X. A survey on wireless mesh networks. Communications Magazine, IEEE Sept 2005; 43(9):S23-S30. DOI: 10.1109/MCOM.2005.1509968.

3. Hossain E, Leung KK (eds). Wireless Mesh Networks: Architectures and Protocols. Springer-Verlag New York, Inc.: Secaucus, NJ, USA, 2010.

4. Zhang Y, Luo J, Hu H (eds). Wireless Mesh Networks: Architectures, Protocols and Standards. Auerbach Publications: Boca Raton, FL, 2007.

5. Misra S, Misra SC, Woungang I. Guide to Wireless Mesh Networks. Springer Publishing Company, Incorporated, 2009.

6. Akyildiz I, Wang X. Wireless Mesh Networks (Advanced Texts in Communications and Networking). John Wiley \& Sons: West Sussex, UK, 2009.

7. Methley S. Essentials of Wireless Mesh Networks. Cambridge University Press: Cambridge, UK, 2009.

8. Aggelou G. Wireless Mesh Networking. McGraw-Hill Professional: Portland, OR, 2008.

9. The Network Simulator NS-2. (Available from: http://www.isi.edu/nsnam/ns/) [accessed on February 20, 2011$].$

10. The ns-3 network simulator2. (Available from: http://www.nsnam.org/) [accessed on February 20, 2011].

11. Qualnet Simulator. (Available from: www.scalable-networks.com) [accessed on February 20, 2011].

12. Zeng X, Bagrodia R, Gerla M. Glomosim: A library for parallel simulation of large-scale wireless networks. Workshop on Parallel and Distributed Simulation, 1998.

13. OPNET Simulator. (Available from: http://www.opnet.com) [accessed on February 20, 2011].

14. Sobeih A, Hou JC. Simulation framework for sensor networks in jsim. Technical Report UIUCDCS-R-2003-2386, Nov. 2003.

15. Newport C, Kotz D, Yuan Y, Gray RS, Liu J, Elliott C. Experimental evaluation of wireless simulation assumptions. SIMULATION: Transactions of The Society for Modeling and Simulation International September 2007; 83(9):643-661.

16. Kashyap A, Ganguly S, Das SR. Measurement-based approaches for accurate simulation of 802.11-based wireless networks. In Proceedings of the 11th International Symposium on Modeling, Analysis and Simulation of Wireless and Mobile Systems, MSWiM '08. ACM: New York, NY, USA, 2008; 54-59, DOI: 10.1145/1454503.1454516.

17. Kashyap A. Measurement-based modeling of interference in wi-fi networks: techniques and applications. $P h D$ Thesis, Stony Brook, NY, USA, 2008. AAI3386251.

18. Stojmenovic I. Simulations in wireless sensor and ad hoc networks: matching and advancing models, metrics, and solutions. Communications Magazine, IEEE December 2008; 46(12):102-107. DOI: 10.1109/MCOM.2008.4689215.

19. Ben Hamida E, Chelius G, Gorce JM. Impact of the Physical Layer Modeling on the Accuracy and Scalability of Wireless Network Simulation. Simulation 09 2009; 85:574-588. DOI: 10.1177/0037549709106633.

20. Güneş M, Wenig M, Zimmermann A. Realistic mobility and propagation framework for manet simulations. In NETWORKING 2007. Ad Hoc and Sensor Networks, Wireless Networks, Next Generation Internet, Lecture Notes in Computer Science, Vol. 4479, Akyildiz I, Sivakumar R, Ekici E, Oliveira J, McNair J (eds). Springer Berlin: Heidelberg, 2007; 97-107.

21. Durmaz Incel O, Jansen P. Characterization of multi-channel interference. Modeling and Optimization in Mobile, Ad Hoc, and Wireless Networks and Workshops, 2008. WiOPT 2008. 6th International Symposium on, 2008; 429-435, DOI: 10.1109/WIOPT.2008.4586102.

22. Herms A, Lukas G, Ivanov S. Realism in design and evaluation of wireless routing protocols. Proceedings of First International Workshop on Mobile Services and Personalized Environments (MSPE06), 2006.

23. Takai M, Martin J, Bagrodia R. Effects of wireless physical layer modeling in mobile ad hoc networks. In Proceedings of the 2nd ACM international symposium on Mobile ad hoc networking \& computing, MobiHoc '01. ACM: New York, NY, USA, 2001; 87-94, DOI: 10.1145/501426.501429.

24. Conti M, Giordano S. Multihop ad hoc networking: The theory. Communications Magazine, IEEE April 2007; 45(4):78-86. DOI: 10.1109/MCOM.2007.343616.

25. Tropos networks. (Available from: http://www.tropos.com) [accessed on February 20, 2011].

26. Open-mesh. (Available from: http://www.open-mesh.com) [accessed on February 20, 2011].

27. Gupta P, Kumar PR. The capacity of wireless networks. IEEE Transactions on Information Theory 2000; 46(2):388-404

28. Li J, Blake C, De Couto DS, Lee HI, Morris R. Capacity of ad hoc wireless networks. In Proceedings of the 7th Annual International Conference on Mobile Computing and Networking, MobiCom '01. ACM: New York, NY, USA, 2001; 61-69, DOI: 10.1145/381677.381684.

29. Public Safety Statement of Requirements (SoR), April 2006. (Available from: http://www.safecomprogram.gov/) [accessed on February 20, 2011].

30. Miller LE. NIST Wireless Communication Technologies Group, Public Safety Comunications Bibliography accessed 2008. (Available from: http://w3.antd.nist.gov/wctg/manet/safetybib.html) [accessed on February 20, 2011].

31. Portmann M, Pirzada AA. Wireless mesh networks for public safety and crisis management applications. IEEE Internet Computing 2008; 12(1):18-25. DOI: 10.1109/MIC.2008.25. 
32. Balachandran K, Budka KC, Chu TP, Doumi TL, Kang JH. Mobile responder communication networks for public safety. Communications Magazine, IEEE Jan 2006; 44(1):56-64. DOI: 10.1109/MCOM.2006.1580933.

33. FCC Second Report and Order and Further Notice of Proposed Rulemaking in the matter of the $4.9 \mathrm{GHz}$ Band Transferred from Federal Use. (Available from: http://wireless.fcc.gov/releases/fcc0247.pdf) [accessed on February 20, 2011].

34. Flickenger R. Building Wireless Community Community Networks. O’Reilly \& Associates, Inc: Sebastopol, CA, USA, 2003.

35. Biswas S, Tatchikou R, Dion F. Vehicle-to-vehicle wireless communication protocols for enhancing highway traffic safety. Communications Magazine, IEEE Jan 2006; 44(1):74-82. DOI: 10.1109/MCOM.2006.1580935.

36. The zigbee alliance. (Available from: http://www.zigbee.org/) [accessed on February 20, 2011].

37. The wimax forum. (Available from: http://www.wimaxforum.org/) [accessed on February 20, 2011].

38. Scalabrino N, De Pellegrini F, Riggio R, Maestrini A, Costa C, Chlamtac I. Measuring the quality of voip traffic on a wimax testbed. Testbeds and Research Infrastructure for the Development of Networks and Communities, 2007. TridentCom 2007. 3rd International Conference on, 2007; 1-10, DOI: 10.1109/TRIDENTCOM.2007.4444719.

39. Mignanti S, Castellano M, Spada M, Simoes P, Tamea G, Cimmino A, Neves PM, Marchetti I, Andreotti F, Landi G, et al. Weird testbeds with fixed and mobile wimax technology for user applications, telemedicine and monitoring of impervious areas. In Proceedings of the 4th International Conference on Testbeds and research infrastructures for the development of networks \& communities, TridentCom '08. ICST (Institute for Computer Sciences, Social-Informatics and Telecommunications Engineering): ICST, Brussels, Belgium, Belgium, 2008; 18:1-18:10. (Available from: http://portal.acm.org/citation.cfm?id=1390576.1390598) [accessed on February 20, 2011].

40. Westall J, Martin J. Performance characteristics of an operational wimax network. Mobile Computing, IEEE Transactions on December 2010; PP(99):1. DOI: 10.1109/TMC.2010.226.

41. The status of IEEE 802.11s standard. (Available from: http://grouper.iee.org/groups/802/11/Reports/tgs_update. htm) [accessed on February 20, 2011].

42. Wang X, Lim AO. Ieee 802.11s wireless mesh networks: Framework and challenges. Ad Hoc Networks 2008; 6(6):970-984. DOI: 10.1016/j.adhoc.2007.09.003.

43. Lin Y-D, Tsao S-L, Chang S-L, Cheng S-Y, Ku C-Y. Design issues experimental studies of wireless lan mesh. Wireless Communications, IEEE April 2010; 17(2):32-40. DOI: 10.1109/MWC.2010.5450658.

44. Camp J, Knightly E. The ieee 802.11s extended service set mesh networking standard. Communications Magazine, IEEE August 2008; 46(8):120-126. DOI: 10.1109/MCOM.2008.4597114.

45. Garroppo RG, Giordano S, Tavanti L. Implementation frameworks for ieee 802.11s systems. Computer Communications 2010; 33(3):336-349. DOI: 10.1016/j.comcom.2009.10.001. (Available form: http://www.sciencedirect. com/science/article/B6TYP-4XFGJCS-2/2/7a5080efe52a20994131b4c7232368e2) [accessed on February 20, 2011].

46. Bahr M. Update on the Hybrid Wireless Mesh Protocol of IEEE 802.11s. Mobile Adhoc and Sensor Systems, 2007. MASS 2007. IEEE Internatonal Conference on, 2007; 1-6, DOI: 10.1109/MOBHOC.2007.4428721.

47. Ieee draft standard for information technology-telecommunications and information exchange between systemslocal and metropolitan area networks-specific requirements part 11: Wireless lan medium access control (mac) and physical layer (phy) specifications amendment 10: Mesh networking. IEEE Unapproved Draft Std P802.11s/D4.0, Dec 2009, December 2009, DOI: 10.1109/IEEESTD.2009.5399265.

48. Ad M, Royer EM, Perkins CE, Das SR. Ad hoc on-demand distance vector (aodv) routing 1999. (Available from: http://citeseerx.ist.psu.edu/viewdoc/summary?doi=10.1.1.47.2139) [accessed on February 20, 2011].

49. Johnson DB, Maltz DA, Hu YC. The dynamic source routing protocol for mobile ad hoc networks (dsr). Technical Report, IETF MANET Working Group, February 2007. (Availabel from: http://tools.ietf.org/html/rfc4728) [accessed on February 20, 2011].

50. Clausen T, Jacquet P. Optimized Link State Routing Protocol (OLSR). RFC 3626 (Experimental), October 2003. (Available from: http://www.ietf.org/rfc/rfc3626.txt) [accessed on February 20, 2011].

51. Campista MEM, Esposito PM, Moraes IM, Costa LHMK, Duarte OCMB, Passos DG, de Albuquerque CVN, Saade DCM, Rubinstein MG. Routing metrics, protocols for wireless mesh networks. Network, IEEE jan-feb 2008; 22(1):6-12. DOI: 10.1109/MNET.2008.4435897.

52. Yang Y, Wang J, Kravets R. Designing routing metrics for mesh networks. Proceedings of the IEEE Workshop on Wireless Mesh Networks (WiMesh). IEEE Press, 2005.

53. Koksal CE. Quality-aware routing metrics in wireless mesh networks. In Wireless Mesh Networks, Hossain E, Leung K (eds). Springer: US, 2007; 227-243.

54. Parissidis G, Karaliopoulos M, Baumann R, Spyropoulos T, Plattner B. Routing metrics for wireless mesh networks. In Guide to Wireless Mesh Networks, Misra S, Misra SC, Woungang I (eds). Computer Communications and Networks, Springer: London, 2009; 199-230.

55. Yang Y, Wang J. Design guidelines for routing metrics in multihop wireless networks. INFOCOM 2008. The 27th Conference on Computer Communications. IEEE, 2008; 1615-1623, DOI: 10.1109/INFOCOM.2008.222.

56. De Couto DSJ, Aguayo D, Bicket J, Morris R. A high-throughput path metric for multi-hop wireless routing. In MobiCom '03: Proceedings of the 9th annual international conference on Mobile computing and networking. ACM: New York, NY, USA, 2003; 134-146, DOI: 10.1145/938985.939000. 
57. Draves R, Padhye J, Zill B. Routing in multi-radio, multi-hop wireless mesh networks. In MobiCom '04: Proceedings of the 10th annual international conference on Mobile computing and networking. ACM: New York, NY, USA, 2004; 114-128, DOI: 10.1145/1023720.1023732.

58. Yang Y, Wang J, Kravets R. Interference-aware load balancing for multihop wireless networks. Technical Report, University of Illinois at Urbana-Champaign, 2005. (Available from: http://www.cs.uiuc.edu/research/techreports. php?report=UIUCDCS-R-2005-2526) [accessed on February 20, 2011].

59. OpenWrt. Openwrt. (Available from: http://www.openwrt.org/) [accessed on February 20, 2011].

60. Meraki I. Meraki. (Available from: http://www.meraki.com/) [accessed on February 20, 2011].

61. Inc L. locustworld. (Availble from: http://locustworld.com/) [accessed on February 20, 2011].

62. Microsoft. Self organizing wireless mesh networks. (Available from: http://research.microsoft.com/en-us/projects/ mesh/) [accessed on February 20, 2011].

63. Chavoutier V, Maniezzo D, Palazzi CE, Gerla M. Multimedia over wireless mesh networks: Results from a real testbed evaluation. Proceedings of Mediteranean Ad hoc Networking Workshop, 2007.

64. Hyacinth: An ieee 802.11-based multi-channel wireless mesh network. URL http://www.ecsl.cs.sunysb.edu/ multichannel/ [accessed on February 20, 2011].

65. Raniwala A, Gopalan K, Chiueh T. Centralized channel assignment and routing algorithms for multi-channel wireless mesh networks. ACM SIGMOBILE Mobile Computing and Communications Review 2004; 8(2):50-65. DOI: 10.1145/997122.997130.

66. Skalli H, Ghosh S, Das S, Lenzini L, Conti M. Channel assignment strategies for multiradio wireless mesh networks: Issues and solutions. Communications Magazine, IEEE November 2007; 45(11):86-95. DOI: 10.1109/MCOM.2007.4378326.

67. Draves R, Padhye J, Zill B. Routing in multi-radio, multi-hop wireless mesh networks. In MobiCom '04: Proceedings of the 10th annual international conference on Mobile computing and networking. ACM: New York, NY, USA, 2004; 114-128, DOI: 10.1145/1023720.1023732.

68. Marina MK, Das SR. A topology control approach for utilizing multiple channels in multi-radio wireless mesh networks, Vol. 1, 3-7 Oct 2005; 381-390, DOI: 10.1109/ICBN.2005.1589641.

69. Subramanian AP, Gupta H, Das SR, Cao J. Minimum interference channel assignment in multiradio wireless mesh networks. IEEE Transactions on Mobile Computing 2008; 7(12):1459-1473. DOI: 10.1109/TMC.2008.70.

70. Subramanian AP, Gupta H, Das SR. Minimum interference channel assignment in multi-radio wireless mesh networks. Sensor, Mesh and Ad Hoc Communications and Networks, 2007. SECON '07. 4th Annual IEEE Communications Society Conference on, June 2007; 481-490, DOI: 10.1109/SAHCN.2007.4292860.

71. Mohsenian-Rad AH, Wong VWS. Joint logical topology design, interface, assignment, channel allocation, and routing for multi-channel wireless mesh networks. Wireless Communications, IEEE Transactions on December 2007; 6(12):4432-4440. DOI: 10.1109/TWC.2007.060312.

72. Rad AHM, Wong VWS. Wsn16-4: Logical topology design and interface assignment for multi-channel wireless mesh networks. Global Telecommunications Conference, 2006. GLOBECOM '06. IEEE, Nov 2006; 1-6, DOI: 10.1109/GLOCOM.2006.985.

73. Cho S, kwon Kim C. Interference-Aware Multi-Channel Assignment in Multi-Radio Wireless Mesh Networks. IEICE Transactions 2008; 91-B(5):1436-1445.

74. So J, Vaidya NH. Multi-channel mac for ad hoc networks: handling multi-channel hidden terminals using a single transceiver. In MobiHoc '04: Proceedings of the 5th ACM international symposium on Mobile ad hoc networking and computing. ACM: New York, NY, USA, 2004; 222-233, DOI: 10.1145/989459.989487.

75. Bahl P, Chandra R, Dunagan J. Ssch: slotted seeded channel hopping for capacity improvement in ieee 802.11 ad-hoc wireless networks. In MobiCom '04: Proceedings of the 10th annual international conference on Mobile computing and networking. ACM: New York, NY, USA, 2004; 216-230, DOI: 10.1145/1023720.1023742.

76. Raniwala A, Chiueh T. Evaluation of a wireless enterprise backbone network architecture. High Performance Interconnects, 2004. Proceedings. 12th Annual IEEE Symposium on, Aug 2004; 98-104, DOI: 10.1109/CONECT.2004.1375211.

77. Raniwala A, Chiueh T. Architecture and algorithms for an ieee 802.11-based multi-channel wmn. IEEE INFOCOM '05, Mar 2005, DOI: 10.1109/INFCOM.2005.1498497.

78. Kyasanur P, Vaidya NH. Routing and interface assignment in multi-channel multi-interface wireless networks. In Wireless Communications and Networking Conference, 2005 IEEE, Vol. 4, 13-17 March 2005; 2051-2056, DOI: 10.1109/WCNC.2005.1424834.

79. Kyasanur P, Vaidya NH. Routing and link-layer protocols for multi-channel multi-interface ad hoc wireless networks. ACM SIGMOBILE Mobile Computing and Communications Review 2006; 10(1):31-43. DOI: 10.1145/1119759.1119762.

80. Ramachandran KN, Belding EM, Almeroth KC, Buddhikot MM. Interference-aware channel assignment in multi-radio wireless mesh networks. IEEE INFOCOM '06, Apr 2006; 1-12, DOI: 10.1109/INFOCOM.2006.177.

81. Plummer A, Wu T, Biswas S. A cognitive spectrum assignment protocol using distributed conflict graph construction. IEEE MILCOM, 2007; 1-7.

82. MIT-RoofNet. roofnet[mit roofnet]. (Available from: http://pdos.csail.mit.edu/roofnet/doku.php) [accessed on February 20, 2011]. 
83. RoofNet B. Berlin roofnet - sarwiki. (Available from: http://sarwiki.informatik.hu-berlin.de/BerlinRoofNet) [accessed on February 20, 2011].

84. Camp J, Robinson J, Steger C, Knightly E. Measurement driven deployment of a two-tier urban mesh access network. In MobiSys '06: Proceedings of the 4th international conference on Mobile systems, applications and services. ACM: New York, NY, USA, 2006; 96-109, DOI: 10.1145/1134680.1134691.

85. Lan Kc, Wang Z, Hassan M, Moors T, Berriman R, Libman L, Ott M, Landfeldt B, Zaidi Z. Experiences in deploying a wireless mesh network testbed for traffic control. ACM SIGCOMM Computer Communication Review 2007; 37(5):17-28. DOI: 10.1145/1290168.1290171.

86. Purdue university wireless mesh testbed. (Available from: https://engineering.purdue.edu/MESH) [accessed on February 20, 2011].

87. Wu D, Gupta D, Liese S, Mohapatra P. Qurinet: quail ridge natural reserve wireless mesh network. In WiNTECH '06: Proceedings of the 1st international workshop on Wireless network testbeds, experimental evaluation \& characterization. ACM: New York, NY, USA, 2006; 109-110, DOI: 10.1145/1160987.1161015.

88. Ishmael J, Bury S, Pezaros D, Race N. Deploying rural community wireless mesh networks. Internet Computing, IEEE July-Aug 2008; 12(4):22-29. DOI: 10.1109/MIC.2008.76.

89. Allen W, Martin A, Rangarajan A. Designing and deploying a rural ad-hoc community mesh network testbed. Local Computer Networks, 2005. 30th Anniversary. The IEEE Conference on, 2005; 4 pp.-743, DOI: 10.1109/LCN.2005.51.

90. Tsarmpopoulos N, Kalavros I, Lalis S. A low-cost and simple-to-deploy peer-to-peer wireless network based on open source linux routers. In TRIDENTCOM '05: Proceedings of the First International Conference on Testbeds and Research Infrastructures for the DEvelopment of NeTworks and COMmunities. IEEE Computer Society: Washington, DC, USA, 2005; 92-97, DOI: 10.1109/TRIDNT.2005.3.

91. RuralNet. Ruralnet (digital gangetic plains: Dgp) 802.11-based low-cost networking for rural india. (Available from: http://www.cse.iitk.ac.in/users/braman/dgp.html) [accessed on February 20, 2011].

92. CUWiN. Cuwin - community wireless. (Available from: http://www.cuwireless.net/) [accessed on February 20, 2011].

93. Serrano P, de la Oliva A, Bernardos CJ, Soto I, Banchs A, Azcorra A. A carmen mesh experience: deployment and results. World of Wireless, Mobile and Multimedia Networks \& Workshops, 2009. WoWMoM 2009. IEEE International Symposium on a, 2009; 1-6, DOI: 10.1109/WOWMOM.2009.5282418.

94. Passos D, Teixeira DV, Muchaluat-saade DC, Magalhes LCS, Albuquerque CVN. Mesh network per formance measurements. In 5th International Information and Telecommunication Technologies Symposium, 2006, 2006.

95. Mase K, Owada Y, Okada H, Imai T. A testbed-based approach to develop layer 3 wireless mesh network protocols. In TridentCom '08: Proceedings of the 4th International Conference on Testbeds and research infrastructures for the development of networks \& communities. ICST (Institute for Computer Sciences, Social-Informatics and Telecommunications Engineering): ICST, Brussels, Belgium, Belgium, 2008; 1-6.

96. Iqbal M, Wang X, Wertheim D, Zhou X. Swanmesh: A multicast enabled dual-radio wireless mesh network for emergency and disaster recovery services. Journal of Communications 2009; 4(5):298-306.

97. Capone A, Cesana M, Napoli S, Pollastro A. Mobimesh: a complete solution for wireless mesh networking. Mobile Adhoc and Sensor Systems, 2007. MASS 2007. IEEE Internatonal Conference on, 2007; 1-3, DOI: 10.1109/MOBHOC.2007.4428698.

98. Lee J, Lee SJ, Kim W, Jo D, Kwon T, Choi Y. Rss-based carrier sensing and interference estimation in 802.11 wireless networks. Sensor, Mesh and Ad Hoc Communications and Networks, 2007. SECON '07. 4th Annual IEEE Communications Society Conference on, 2007; 491-500, DOI: 10.1109/SAHCN.2007.4292861.

99. Lundgren H, Ramachandran K, Belding-Royer E, Almeroth K, Benny M, Hewatt A, Touma A, Jardosh A. Experiences from the design, deployment, and usage of the ucsb meshnet testbed. Wireless Communications, IEEE April 2006; 13(2):18-29. DOI: 10.1109/MWC.2006.1632477.

100. UMIC-Meshnet. What is umic-mesh.net? (Available from: http://www.umic-mesh.net/home/) [accessed on February 20, 2011].

101. Amir Y, Danilov C, Musaloiu-Elefteri R, Rivera N. The smesh wireless mesh network. Technical Memorandum CNDS-2009-3, Johns Hopkins University, Boeing Phantom Works, April 2009.

102. SMesh. Smesh. (Available from: http://www.smesh.org/) [accessed on February 20, 2011].

103. Leipzig wireless mesh testbed. (Available from: http://rvs.informatik.uni-leipzig.de/en/forschung/testbed.php) [accessed on February 20, 2011].

104. Kim KH, Shin KG. Self-healing multi-radio wireless mesh networks. In MobiCom '07: Proceedings of the 13th annual ACM international conference on Mobile computing and networking. ACM: New York, NY, USA, 2007; 326-329, DOI: 10.1145/1287853.1287896.

105. Raniwala A, Chiueh T. Architecture and algorithms for an ieee 802.11-based multi-channel wireless mesh network. In INFOCOM 2005. 24th Annual Joint Conference of the IEEE Computer and Communications Societies. Proceedings IEEE, Vol. 3, 2005; 2223-2234, DOI: 10.1109/INFCOM.2005.1498497.

106. Edmonds C, Joiner D, Springer S, Stephen K, Hamdaoui B. Cognitive wireless mesh network testbed. Wireless Communications and Mobile Computing Conference, 2008. IWCMC '08. International, 2008; 373-376, DOI: 10.1109/IWCMC.2008.65. 
107. Iannone L, Kabassanov K, Fdida S. The meshdvnet wireless mesh network test-bed. In WiNTECH '06: Proceedings of the 1st international workshop on Wireless network testbeds, experimental evaluation \& characterization. ACM: New York, NY, USA, 2006; 107-108, DOI: 10.1145/1160987.1161014.

108. "cnri wireless mesh testbed". (Available from: http://www.cnri.dit.ie/research.mesh.testbed.html/) [accessed on February 20, 2011].

109. Orbit. (Available from: http://www.orbit-lab.org/) [accessed on February 20, 2011].

110. Rice university warp - wireless open-access research platform. (Available from: http://warp.rice.edu/) [accessed on February 20, 2011].

111. Wireless mesh network at carleton university. (Available from: http://kunz-pc.sce.carleton.ca/MESH/index.htm) [accessed on February 20, 2011].

112. Su Y, Gross T. Validation of a miniaturized wireless network testbed. In WiNTECH '08: Proceedings of the third ACM international workshop on Wireless network testbeds, experimental evaluation and characterization. ACM: New York, NY, USA, 2008; 25-32, DOI: 10.1145/1410077.1410084.

113. The pre-ieee $802.11 \mathrm{~s}$ wireless mesh network testbed at auburn university. (Available from: http://www.eng.auburn. edu/users/abidmoh/) [accessed on February 20, 2011].

114. De P, Raniwala A, Krishnan R, Tatavarthi K, Modi J, Syed NA, Sharma S, Chiueh T. Mint-m: an autonomous mobile wireless experimentation platform. In MobiSys '06: Proceedings of the 4th international conference on Mobile systems, applications and services. ACM: New York, NY, USA, 2006; 124-137, DOI: 10.1145/1134680.1134694.

115. Robinson J, Papagiannaki K, Diot C, Guo X, Krishnamurthy L. Experimenting with a multi-radio mesh networking testbed. Proceedings of the First Workshop on Wireless Network Measurements (WiNMee 2005), Trentino, Italy, 2005. (Available from: http://www.winmee.org/2005/papers/WiNMee_Robinson.pdf) [accessed on February 20, 2011].

116. Shrivastava V, Rayanchu S, Yoonj J, Banerjee S. 802.11n under the microscope. In IMC '08: Proceedings of the 8th ACM SIGCOMM conference on Internet measurement. ACM: New York, NY, USA, 2008; 105-110, DOI: $10.1145 / 1452520.1452533$.

117. Pelechrinis K, Broustis I, Salonidis T, Krishnamurthy SV, Mohapatra P. Design and deployment considerations for high performance mimo testbeds. In WICON '08: Proceedings of the 4th Annual International Conference on Wireless Internet. ICST (Institute for Computer Sciences, Social-Informatics and Telecommunications Engineering): ICST, Brussels, Belgium, Belgium, 2008; 1-9.

118. Ramachandran K, Belding-Royer E, AImeroth K. Damon: a distributed architecture for monitoring multi-hop mobile networks. Sensor and Ad Hoc Communications and Networks, 2004. IEEE SECON 2004. 2004 First Annual IEEE Communications Society Conference on, 2004; 601-609, DOI: 10.1109/SAHCN.2004.1381963.

119. Capone A, Cesana M, Napoli S, Pollastro A. Mobimesh: a complete solution for wireless mesh networking. Mobile Adhoc and Sensor Systems, 2007. MASS 2007. IEEE Internatonal Conference on, 2007; 1-3, DOI: 10.1109/MOBHOC.2007.4428698.

120. Riggio R, Scalabrino N, Miorandi D, Chlamtac I. Janus: A framework for distributed management of wireless mesh networks. Testbeds and Research Infrastructure for the Development of Networks and Communities, 2007. TridentCom 2007. 3rd International Conference on, 2007; 1-7, DOI: 10.1109/TRIDENTCOM.2007.4444703.

121. Aseeja V, Zheng R. Meshman: A management framework for wireless mesh networks. Integrated Network Management, 2009. IM '09. IFIP/IEEE International Symposium on, 2009; 226-233, DOI: 10.1109/INM.2009.5188814.

122. Duarte J, Passos D, Valle R, Oliveira E, Muchaluat-Saade D, Albuquerque C. Management issues on wireless mesh networks. Network Operations and Management Symposium, 2007. LANOMS 2007. Latin American, 2007; 8-19, DOI: 10.1109/LANOMS.2007.4362455.

123. Judd G, Steenkiste P. Repeatable and realistic wireless experimentation through physical emulation. SIGCOMM Comput. Commun. Rev. January 2004; 34:63-68. DOI: 10.1145/972374.972386.

124. Bialkowski K, Portmann M. Design of testbed for wireless mesh networks. Antennas and Propagation Society International Symposium (APSURSI), 2010 IEEE, 2010; 1-4, DOI: 10.1109/APS.2010.5562247.

125. Kiess W, Tarp A, Mauve M. On the Topological Repeatability of Experiments with Wireless Multihop Networks. MSWiM 2008: Proceedings of the 11th ACM/IEEE International Conference on Modeling, Analysis and Simulation of Wireless and Mobile Systems, 2008.

126. De P, Raniwala A, Sharma S, Chiueh T. Design considerations for a multihop wireless network testbed. Communications Magazine, IEEE oct 2005; 43(10):102-109. DOI: 10.1109/MCOM.2005.1522132.

127. Burchfield R, Nourbakhsh E, Dix J, Sahu K, Venkatesan S, Prakash R. Rf in the jungle: Effect of environment assumptions on wireless experiment repeatability. Communications, 2009. ICC '09. IEEE International Conference on, 2009; 1-6, DOI: 10.1109/ICC.2009.5199421.

128. Tcpdump/libpcap public repository. (Available from: http://www.tcpdump.org) [accessed on February 20, 2011].

129. Wireshark. (Available from: http://www.wireshark.org) [accessed on February 20, 2011].

130. De P, Raniwala A, Sharma S, Chiueh T. Mint: a miniaturized network testbed for mobile wireless research. In INFOCOM 2005, Vol. 4, 2005; 2731-2742, DOI: 10.1109/INFCOM.2005.1498556.

131. Crossbow Mica Motes. (Available from: http://www.xbow.com) [accessed on February 20, 2011]. 
132. Levis P, Lee N, Welsh M, Culler D. Tossim: accurate and scalable simulation of entire tinyos applications. In Proceedings of the 1st international conference on Embedded networked sensor systems, SenSys '03. ACM: New York, NY, USA, 2003; 126-137.

133. Kawadia V, Kumar P. A cautionary perspective on cross-layer design. Wireless Communications, IEEE Feb 2005; 12(1):3-11. DOI: 10.1109/MWC.2005.1404568.

\section{AUTHORS' BIOGRAPHIES}

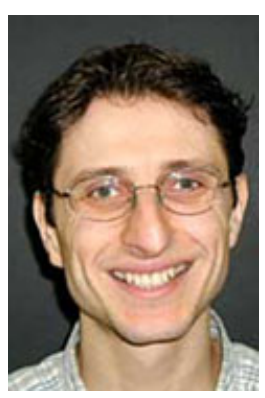

Suleyman Uludag received the $\mathrm{PhD}$ degree from DePaul University in 2007. He is an assistant professor at the University of Michigan-Flint since 2006. His research interests include guaranteed and stochastic routing in wired, wireless mesh and sensor networks, topology aggregation, networking infrastructure in smart grid, and channel assignment in wireless mesh networks. He is a member of the ACM and IEEE.

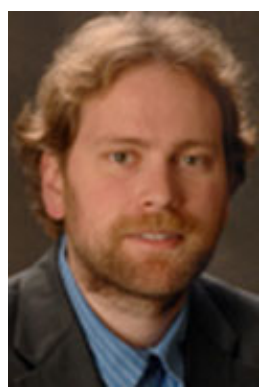

Tom Imboden is an Assistant Professor in the School of Information Systems and Applied Technologies at Southern Illinois University. Mr. Imboden has taught undergraduate networking and security courses at SIUC since 2008 and is pursuing a Ph.D. in Computer Science. Before beginning his career in teaching, Mr. Imboden was employed as a network and systems administrator at several private companies. He completed his B.S. and M.S. from DePaul University in 2003 and 2007, respectively. His research interests include wireless mesh networking, network monitoring protocols, and the use of virtualization technologies in security education.

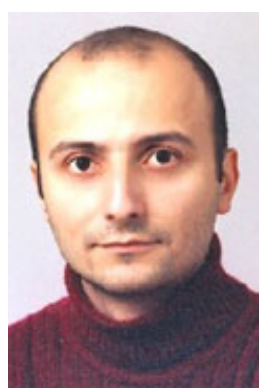

Kemal Akkaya received his BS and MS degrees in Computer Science from Bilkent University, Ankara, Turkey in 1997 and Middle East Technical University (METU), Ankara, Turkey in 1999 respectively. He worked as a software developer in 2000, Ankara, Turkey. $\mathrm{He}$ received his PhD in Computer Science at University of Maryland Baltimore County in 2005. Currently, he is an assistant professor in the Department of Computer Science at Southern Illinois University Carbondale and leads the Advanced Wireless and Sensor Networking Lab. Dr. Akkaya is the Associate Editor of Elsevier Ad Hoc Networks Journal. He has served as the guest editor for Journal of High Speed Networks and Elsevier Computer Communications Journal. He was the workshop chair for the First International Workshop on Smart Grid Networking Infrastructure 2010. He has been the Editorial Liaison Chair for IEEE LCN Conference 2010. Currently, he is the program chair for the First Workshop on Federated Wireless Sensor Systems and publicity chair for IEEE LCN 2011. Dr. Akkaya has also been serving in the TPC of many leading wireless networking conferences including IEEE ICC, Globecom, LCN and WCNC. His research interests include energy aware routing, security and quality of service issues in ad hoc wireless, sensor, mesh and underwater networks. He is the recipient of the Top Cited Article Award from Elsevier Ad Hoc Networks Journal for 2010. Dr. Akkaya is a member of IEEE. 